\title{
ON THE AXIOMATIZABILITY OF IMPOSSIBLE FUTURES
}

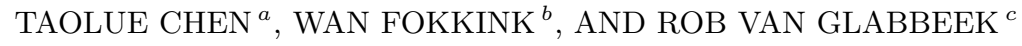 \\ ${ }^{a}$ Middlesex University London, Department of Computer Science, The Burroughs, London NW4 \\ 4BT, United Kingdom \\ e-mail address: t.chen@mdx.ac.uk \\ ${ }^{b}$ VU University Amsterdam, Department of Computer Science, De Boelelaan 1081a, 1081 HV \\ Amsterdam, The Netherlands \\ e-mail address: w.j.fokkink@vu.nl \\ ${ }^{c}$ NICTA, Locked Bag 6016, Sydney, NSW 1466, Australia, and The University of New South Wales, \\ School of Computer Science and Engineering, Sydney, NSW 2052, Australia \\ e-mail address: rvg@cs.stanford.edu
}

\begin{abstract}
A general method is established to derive a ground-complete axiomatization for a weak semantics from such an axiomatization for its concrete counterpart, in the context of the process algebra BCCS. This transformation moreover preserves $\omega$-completeness. It is applicable to semantics at least as coarse as impossible futures semantics. As an application, ground- and $\omega$-complete axiomatizations are derived for weak failures, completed trace and trace semantics. We then present a finite, sound, ground-complete axiomatization for the concrete impossible futures preorder, which implies a finite, sound, ground-complete axiomatization for the weak impossible futures preorder. In contrast, we prove that no finite, sound axiomatization for BCCS modulo concrete and weak impossible futures equivalence is ground-complete. If the alphabet of actions is infinite, then the aforementioned groundcomplete axiomatizations are shown to be $\omega$-complete. If the alphabet is finite, we prove that the inequational theories of BCCS modulo the concrete and weak impossible futures preorder lack such a finite basis.
\end{abstract}

\section{INTRODUCTION}

Labeled transition systems constitute a fundamental model of concurrent computation. Processes are captured by explicitly describing their states and the transitions from state to state together with the actions that produce these transitions. A wide range of notions of behavioral semantics have been proposed, with the aim to identify those states that afford the same observations. Notably, van Glabbeek G01 presented the "linear time - branching time

2012 ACM CCS: [Theory of computation]: Models of computation-Concurrency-Process calculi.

Key words and phrases: Concurrency Theory, Equational Logic, Impossible Futures, Process Algebra.

${ }^{c}$ NICTA is funded by the Australian Government through the Department of Communications and the Australian Research Council through the ICT Centre of Excellence Program.

DOI:10.2168/LMCS-11(3:17)2015
(C) T. Chen, W. Fokkink, and R. van Glabbeek (c) Creative Commons 


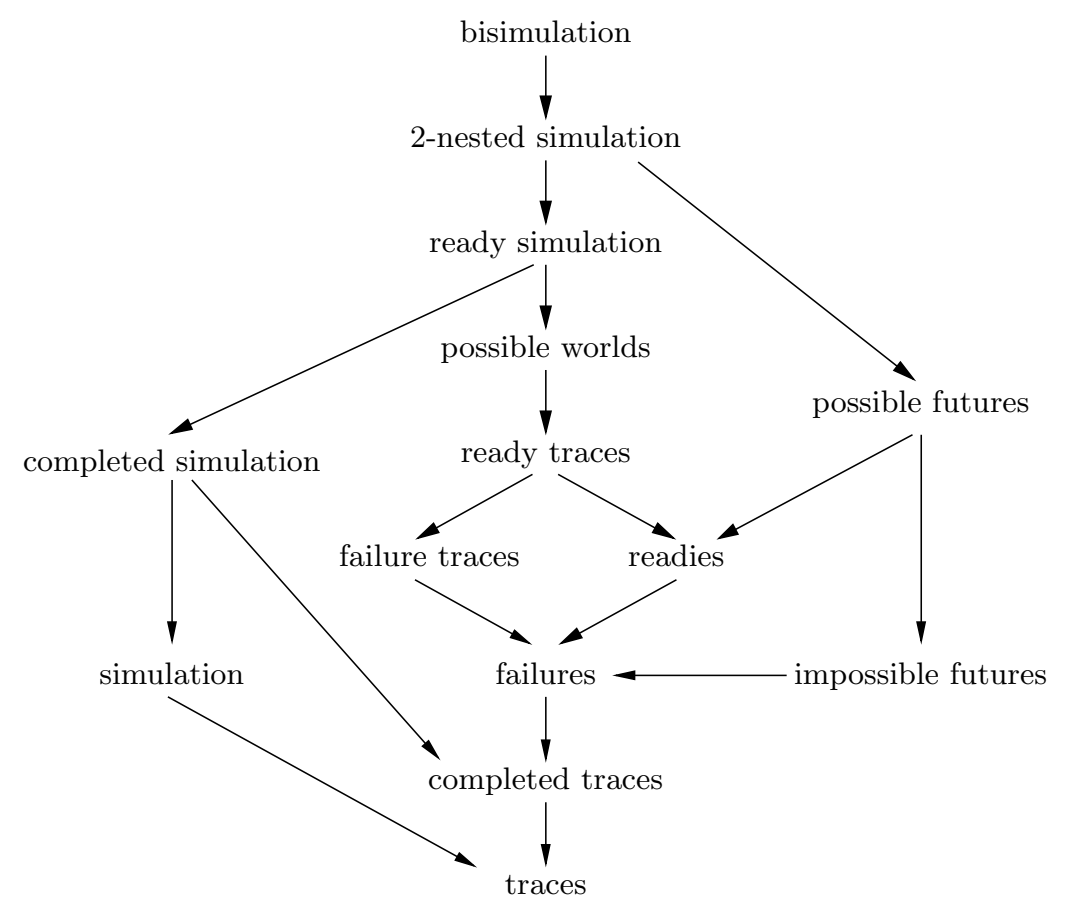

Figure 1: Linear time-branching time spectrum

spectrum I" of behavioral semantics for finitely branching, concrete ${ }^{1}$ sequential processes. These semantics are based on simulation notions or on decorated traces. Fig. 1 depicts this spectrum $\sqrt{2}$ where an arrow from one semantics to another means that the target of the arrow is coarser, i.e. less discriminating, than the source. In G93a, 155 weak semantics, which take into account the hidden action $\tau$, are surveyed. They constitute the "linear time - branching time spectrum II" for finitely branching, abstract, sequential processes.

In this paper, we mainly study impossible futures semantics [V92, VM01, which is a natural variant of possible futures semantics [RB81]. It is also related to fair testing semantics [RV07]. Weak impossible futures equivalence is the coarsest congruence with respect to choice and parallel composition that contains weak bisimilarity with explicit divergence, respects deadlock/livelock traces, and assigns unique solutions to recursive equations [GV06].

The process algebra BCCSP plays a fundamental role in the study of concrete semantics. It contains only basic process algebraic operators from CCS and CSP, but is sufficiently powerful to express all finite synchronization trees (without $\tau$-transitions). Van Glabbeek G01 associated with most behavioral equivalences in his spectrum a sound axiomatization, to equate closed BCCSP terms that are behaviorally equivalent. These axiomatizations were shown to be ground-complete, meaning that all behaviorally equivalent closed BCCSP terms can be equated. The process algebra BCCS (see, e.g., G97]) extends BCCSP with $\tau$, playing the same role as BCCSP in the research of weak semantics.

\footnotetext{
${ }^{1}$ Concrete processes do not feature the hidden action $\tau$. Abstract processes, with $\tau$, come with strong semantics, treating $\tau$ just as any other action, or weak ones, allowing a degree of abstraction from $\tau$ steps.

${ }^{2}$ Impossible futures semantics was missing in the original spectrum I [G01], because it was studied seriously only from 2001 on, the year that G01 appeared.
} 
An $\omega$-complete axiomatization enjoys the property that if all closed instances of an equation can be derived from it, then the equation itself can be derived from it. In universal algebra, such an axiomatization is referred to as a basis for the equational theory of the algebra it axiomatizes. Groote G90 developed a technique of "inverted substitutions" to prove that an axiomatization is $\omega$-complete, and proved for some equivalences in the "linear time - branching time spectrum I" that their equational theory in BCCSP has a finite basis.

In AFIL05, CFLN08, for each preorder and equivalence in the "linear time - branching time spectrum I" it was determined whether a finite, sound, ground-complete axiomatization exists. And if so, whether a finite basis exists for the (in)equational theory. However, for concrete impossible futures semantics the (in)equational theory remained unexplored.

With regard to the axiomatizability of weak semantics, relatively little is known compared to concrete semantics. For some semantics in the "linear time - branching time spectrum II" G93a, a sound and ground-complete axiomatization has been given, in the setting of BCCS (see, e.g., G97]). Moreover, a finite basis has been given for weak, delay, $\eta$ - and branching bisimulation semantics [M89, G93b]. The inequational theory of BCCS modulo the weak impossible futures preorder was studied in [VM01, which offers a finite, sound, ground-complete axiomatization. Voorhoeve and Mauw also proved that their axiomatization is $\omega$-complete. It is worth noting that an infinite alphabet of actions is assumed implicitly VM01, p. 7], because a different action is required for each variable.

The current paper studies the axiomatizability of BCCSP and BCCS for semantics at least as coarse as impossible futures semantics. In summary, we obtain the following results.

(1) A link is established between the axiomatizability of concrete and weak semantics. For any semantics at least as coarse as impossible futures semantics, an algorithm is provided to turn a ground-complete axiomatization of the concrete version into a ground-complete axiomatization of the corresponding weak version. Moreover, if the former axiomatization is $\omega$-complete, then so is the latter.

(2) As an application of this algorithm, we derive finite, sound, ground- and $\omega$-complete axiomatizations for the weak trace, completed trace and failures preorders and equivalences. Failures semantics plays a prominent role in the process algebra CSP [BHR84]. For convergent processes, it coincides with testing semantics [DH84, RV07, and thus is the coarsest congruence for the CCS parallel composition that respects deadlock behavior. A ground-complete axiomatization for weak failures equivalence was already given in G97.

(3) We provide a finite, sound, ground-complete axiomatization for BCCSP modulo the concrete impossible futures preorder $\precsim_{\mathrm{IF}}{ }^{3}$ (By contrast, no such axiomatization exists for the possible futures preorder [AFGI04].) Using (1), a finite, sound, ground-complete axiomatization for the weak impossible futures preorder $\precsim$ WIF is obtained.

(4) We prove that BCCS modulo weak impossible futures equivalence $\simeq_{\mathrm{WIF}}$ does not have a finite, sound, ground-complete axiomatization. Likewise, we prove that BCCSP modulo $\simeq_{\mathrm{IF}}$ does not have a finite, sound, ground-complete axiomatization. The infinite families of equations that we use to prove these negative results are also sound modulo (weak resp. concrete) 2-nested simulation equivalence. Therefore these negative results apply to all BCCS- and BCCSP-congruences that are at least as fine as impossible futures

\footnotetext{
${ }^{3}$ In case of an infinite alphabet of actions, occurrences of action names in axioms should be interpreted as variables, as else most of the axiomatizations would be infinite.
} 
equivalence and at least as coarse as 2-nested simulation equivalence. This infers some results from AFGI04, where among others concrete 2-nested simulation and possible futures equivalence were considered.

(5) We investigate $\omega$-completeness for impossible futures semantics. First, we prove that if the alphabet of actions is infinite, then the aforementioned ground-complete axiomatization for BCCS modulo $\precsim_{\mathrm{IF}}$ is $\omega$-complete. Here we apply the technique of inverted substitutions from G90. Only, that technique was originally developed for equivalences. Therefore, as an aside, we adapt this technique in such a way that it applies to preorders. By (1), this result carries over to $\precsim$ WIF. Second, we prove that in case of a finite alphabet of actions, the inequational theories of BCCS modulo WIF $_{\text {and of BCCSP modulo }}$ IF do not have a finite basis.

To achieve the negative results, we employ what in [AFIL05, Sect. 2.3] is called the prooftheoretic technique. That is, to prove the nonexistence of a finite axiomatization for an equivalence $\equiv$ (resp. preorder $\sqsubseteq$ ), it suffices to provide an infinite family of (in)equations $t_{n} \approx u_{n}$ (resp. $\left.t_{n} \preccurlyeq u_{n}\right)(n=1,2,3, \ldots)$ that are all sound modulo $\equiv$ (resp. $\sqsubseteq$ ), and to associate with every finite set of sound (in)equations $E$ a property $P_{E}$ that holds for all (in)equations derivable from $E$, but does not hold for at least one of the equations $t_{n} \approx u_{n}$ (resp. inequations $t_{n} \preccurlyeq u_{n}$ ). This implies that $t_{n} \approx u_{n}$ (resp. $t_{n} \preccurlyeq u_{n}$ ) is not derivable from $E$. It follows that every finite set of sound (in)equations is necessarily incomplete, so $\equiv$ (resp. Б) lacks a finite axiomatization. On top of this, a saturation principle is introduced, to transform a single summand into a large collection of semi-saturated summands.

Impossible futures semantics is the first example that, in the context of BCCSP/BCCS, affords a ground-complete axiomatization modulo the preorder, while missing a groundcomplete axiomatization modulo the equivalence. This surprising fact suggests that if one wants to show $p \simeq_{\mathrm{IF}} q$, in general one has to resort to deriving $p \precsim$ IF $q$ and $q \precsim$ IF $p$ separately, instead of proving it directly. In AFI07, FGP07 an algorithm is presented which produces from an axiomatization for BCCSP modulo a preorder, an axiomatization for BCCSP modulo the associated equivalence. If the original axiomatization for the preorder is ground-complete or $\omega$-complete, then so is the resulting axiomatization for the equivalence. In [CFG08, we have shown that the same algorithm applies equally well to weak semantics. However, these algorithms apply only to semantics that are at least as coarse as ready simulation semantics. Since impossible futures semantics is incomparable to ready simulation semantics, it falls outside the scope of [AFI07, FGP07, CFG08. Interestingly, our results yield that no such algorithm can exist for impossible futures semantics.

The paper is structured as follows. Sect. 2 presents basic definitions regarding the studied semantics, the process algebra's BCCSP and BCCS, and (in)equational logic. Sect. 3 describes a transformation of an axiomatization for a concrete to an axiomatization for a corresponding weak semantics. In Sect. 4 this transformation is applied to failures, completed trace and trace semantics. Sect. 5 provides finite, sound, ground-complete axiomatizations for $\precsim_{\mathrm{IF}}$ and $\precsim_{\mathrm{WIF}}$; it also presents the aforementioned negative result for $\simeq_{\mathrm{IF}}$ and $\simeq_{\mathrm{WIF}}$. Sect. 6 is devoted to the proofs of the positive and negative results regarding $\omega$-completeness. Sect. 7 concludes the paper.

This paper combines and extends two previous papers in conference proceedings [CF08] and CFG09]. In particular, CF08 dealt with the concrete impossible futures semantics and [CFG09] extended it to weak impossible futures and weak failures semantics. Here, new results are presented in Sect. 3, which yield a much simplified proof for the results regarding 
weak failures semantics given in CFG09, and a unified treatment of concrete and weak impossible futures semantics.

\section{Preliminaries}

2.1. Labeled transition systems. Let $A$ be a nonempty, countable set of concrete (a.k.a. observable, external, visible) actions, which is ranged over by $a, b, c$. The distinguished symbol $\tau$ denotes a hidden (a.k.a. unobservable, internal, invisible) action. We assume that $\tau \notin A$ and write $A_{\tau}$ for $A \cup\{\tau\}$, which is ranged over by $\alpha$.

Definition 2.1. A labeled transition system (LTS) consists of a set of states $S$, with typical element $s$, and a transition relation $\rightarrow \subseteq S \times A_{\tau} \times S$.

We introduce some notation: $s \stackrel{\alpha}{\rightarrow} s^{\prime}$ means $\left(s, \alpha, s^{\prime}\right)$ is an element of $\rightarrow$; by $s \stackrel{\alpha}{\rightarrow}$ we denote that $s \stackrel{\alpha}{\rightarrow} s^{\prime}$ for some $s^{\prime}$, and $s \stackrel{\alpha}{\rightarrow}$ is the negation of this property; for $\alpha_{1} \cdots \alpha_{k}$ a sequence of labels, $s \stackrel{\alpha_{1} \cdots \alpha_{k}}{\rightarrow} s^{\prime}$ means there exist states $s_{0}, \ldots, s_{k}$ such that $s=s_{0} \stackrel{\alpha_{7}}{\rightarrow} \ldots \stackrel{\alpha_{k}}{\rightarrow} s_{k}=s^{\prime}$; the empty sequence is denoted by $\varepsilon$; we define $\mathcal{I}(s)=\left\{\alpha \in A_{\tau} \mid s \stackrel{\alpha}{\rightarrow}\right\}$; we write $\Rightarrow$ for the transitive-reflexive closure of $\stackrel{\tau}{\rightarrow}$, i.e., $\Rightarrow \stackrel{\text { def }}{=}(\stackrel{\tau}{\rightarrow})^{*}$; and $s \Rightarrow \stackrel{\alpha}{\rightarrow}$ means there are two states $s^{\prime}, s^{\prime \prime}$ with $s \Rightarrow s^{\prime} \stackrel{\alpha}{\rightarrow} s^{\prime \prime}$.

\subsection{Decorated trace semantics.}

\section{Definition 2.2.}

- A sequence $a_{1} \cdots a_{k} \in A^{*}$ with $k \geq 0$ is a trace of a state $s$ if there is a state $s^{\prime}$ such that $s \stackrel{a_{1} \cdots a_{k}}{\rightarrow} s^{\prime}$. It is a completed trace of $s$ if moreover $\mathcal{I}\left(s^{\prime}\right)=\emptyset$. We write $\mathcal{T}(s)$ (resp. $\mathcal{C} \mathcal{T}(s)$ ) for the set of traces (resp. completed traces) of state $s$. We write $s_{1} \precsim \mathrm{T} s_{2}$ (resp. $\left.s_{1} \precsim \mathrm{CT} s_{2}\right)$ if $\mathcal{T}\left(s_{1}\right) \subseteq \mathcal{T}\left(s_{2}\right)$ (resp. $\left.\mathcal{C} \mathcal{T}\left(s_{1}\right) \subseteq \mathcal{C} \mathcal{T}\left(s_{2}\right)\right) \bigsqcup^{4}$

- A sequence $a_{1} \cdots a_{k} \in A^{*}$ with $k \geq 0$ is a weak trace of a state $s$ if there is a state $s^{\prime}$ such that $s \Rightarrow \stackrel{a_{1}}{\rightarrow} \Rightarrow \cdots \Rightarrow \stackrel{a_{k}}{\rightarrow} \Rightarrow s^{\prime}$. It is a weak completed trace of $s$ if moreover $\mathcal{I}\left(s^{\prime}\right)=\emptyset$. We write $\mathcal{W} \mathcal{T}(s)$ (resp. $\mathcal{W C} \mathcal{T}(s)$ ) for the set of weak traces (resp. weak completed traces) of $s$. We write $s_{1} \precsim{ }_{\mathrm{WT}} s_{2}$ if $\mathcal{W} \mathcal{T}\left(s_{1}\right) \subseteq \mathcal{W} \mathcal{T}\left(s_{2}\right)$. We write $s_{1} \precsim{ }_{\mathrm{WCT}} s_{2}$ if $\mathcal{W C T}\left(s_{1}\right) \subseteq \mathcal{W C T}\left(s_{2}\right)$ and $s_{1} \stackrel{\tau}{\rightarrow}$ implies that $s_{2} \stackrel{\tau}{\rightarrow}$.

The extra requirement that $s_{1} \stackrel{\tau}{\rightarrow}$ implies $s_{2} \stackrel{\tau}{\rightarrow}$, in the definition of $\precsim$ WCT , is needed to make it a precongruence for the process algebra $\operatorname{BCCS}(A)$ (see Remark 2.5).

\section{Definition 2.3.}

- A pair $\left(a_{1} \cdots a_{k}, B\right)$ with $k \geq 0, a_{1} \cdots a_{k} \in A^{*}$ and $B \subseteq A$ is a failure pair of a state $s$ if there is a state $s^{\prime}$ such that $s \stackrel{a_{1} \cdots a_{k}}{\rightarrow} s^{\prime}$ and $\mathcal{I}\left(s^{\prime}\right) \subseteq A \backslash B$. We write $s_{1} \precsim_{F} s_{2}$ if the failure pairs of $s_{1}$ are also failure pairs of $s_{2}$.

- A pair $\left(a_{1} \cdots a_{k}, B\right)$ with $k \geq 0, a_{1} \cdots a_{k} \in A^{*}$ and $B \subseteq A$ is a weak failure pair of a state $s$ if there is a state $s^{\prime}$ such that $s \Rightarrow \stackrel{a_{1}}{\rightarrow} \Rightarrow \cdots \Rightarrow \stackrel{a_{k}}{\rightarrow} \Rightarrow s^{\prime}$ and $\mathcal{I}\left(s^{\prime}\right) \subseteq A \backslash B$. We write $s_{1} \precsim$ WF $s_{2}$ if the weak failure pairs of $s_{1}$ are also weak failure pairs of $s_{2}$ and $s_{1} \stackrel{\tau}{\rightarrow}$ implies that $s_{2} \stackrel{\tau}{\rightarrow}$.

\footnotetext{
${ }^{4}$ In G01, $s_{1} \precsim \mathrm{CT} s_{2}$ is defined to hold iff $\mathcal{C} \mathcal{T}\left(s_{1}\right) \subseteq \mathcal{C} \mathcal{T}\left(s_{2}\right)$ and $\mathcal{T}\left(s_{1}\right) \subseteq \mathcal{T}\left(s_{2}\right)$. Here we can skip the latter condition, as we will work with finite transition systems, where $\mathcal{C} \mathcal{T}\left(s_{1}\right) \subseteq \mathcal{C} \mathcal{T}\left(s_{2}\right)$ implies $\mathcal{T}\left(s_{1}\right) \subseteq \mathcal{T}\left(s_{2}\right)$.
} 


\section{Definition 2.4.}

- A pair $\left(a_{1} \cdots a_{k}, B\right)$ with $k \geq 0, a_{1} \cdots a_{k} \in A^{*}$ and $B \subseteq A^{*}$ is an impossible future of a state $s$ if $s \stackrel{a_{1} \cdots a_{k}}{\rightarrow} s^{\prime}$ for some state $s^{\prime}$ with $\mathcal{T}\left(s^{\prime}\right) \cap B=\emptyset$. We write $s_{1} \precsim$ IF $s_{2}$ if the impossible futures of $s_{1}$ are included in those of $s_{2}$.

- A pair $\left(a_{1} \cdots a_{k}, B\right)$ with $k \geq 0, a_{1} \cdots a_{k} \in A^{*}$ and $B \subseteq A^{*}$ is a weak impossible future of a state $s$ if there is a trace $s \Rightarrow \stackrel{a_{1}}{\rightarrow} \Rightarrow \cdots \Rightarrow \stackrel{a_{k}}{\rightarrow} \Rightarrow s^{\prime}$ with $\mathcal{W} \mathcal{T}\left(s^{\prime}\right) \cap B=\emptyset$. We write $s_{1} \precsim$ WIF $s_{2}$ if the weak impossible futures of $s_{1}$ are also weak impossible futures of $s_{2}$, $\mathcal{W} \mathcal{T}\left(s_{1}\right)=\mathcal{W} \mathcal{T}\left(s_{2}\right)$, and $s_{1} \stackrel{\tau}{\rightarrow}$ implies that $s_{2} \stackrel{\tau}{\rightarrow}$.

The extra requirement that $\mathcal{W} \mathcal{T}\left(s_{1}\right)=\mathcal{W} \mathcal{T}\left(s_{2}\right)$, in the definition of $\precsim$ WIF , is again needed to make it a precongruence for $\operatorname{BCCS}(A)$ (see Remark 2.5).

Given a preorder $\precsim_{R}$, the associated equivalence is denoted with $\simeq_{R}$, where $s_{1} \simeq_{R} s_{2}$ if both $s_{1} \precsim_{R} s_{2}$ and $s_{2} \precsim_{R} s_{1}$.

2.3. Process algebras BCCS and BCCSP. $\operatorname{BCCS}(A)$ is a basic process algebra for expressing finite process behaviors. Its signature consists of the constant $\mathbf{0}$, the binary operator ${ }_{-}+_{-}$, and unary prefix operators $\alpha_{-}$, where $\alpha$ ranges over $A_{\tau}$. The process algebra $\operatorname{BCCSP}(A)$ is obtained by excluding the prefix operator $\tau_{-}$. In the context of process algebra, $A$ is called the alphabet. Again it is required that $A \neq \emptyset$.

Intuitively, closed $\operatorname{BCCS}(A)$ terms, which are ranged over by $p, q, r$, represent finite process behaviors, where $\mathbf{0}$ does not exhibit any behavior, $p+q$ offers a choice between the behaviors of $p$ and $q$, and $\alpha p$ executes action $\alpha$ to transform into $p$. This intuition is captured by the transition rules below. They give rise to $A_{\tau}$-labeled transitions between closed $\operatorname{BCCS}(A)$ terms. We assume a countably infinite set $V$ of variables; $x, y, z$ denote elements of $V$, ranging over $\operatorname{BCCS}(A)$ terms.

$$
\overline{\alpha x \stackrel{\alpha}{\rightarrow} x} \quad \frac{x \stackrel{\alpha}{\rightarrow} x^{\prime}}{x+y \stackrel{\alpha}{\rightarrow} x^{\prime}} \quad \frac{y \stackrel{\alpha}{\rightarrow} y^{\prime}}{x+y \stackrel{\alpha}{\rightarrow} y^{\prime}}
$$

Open BCCS terms, denoted by $t, u, v, w$, may contain variables from $V$. We write $\operatorname{var}(t)$ for the set of variables occurring in $t$. It is technically convenient to extend the operational semantics to open terms. There are no additional rules for variables, which effectively means that they do not exhibit any behavior.

An occurrence of an action or variable in a term is said to be initial if it is not in the context of a prefix operator.

The depth of a term $t$ is the length of the longest trace of $t$. It is defined inductively as follows: $\operatorname{depth}(\mathbf{0})=\operatorname{depth}(x)=0 ; \operatorname{depth}(\alpha t)=1+\operatorname{depth}(t) ;$ and $\operatorname{depth}(t+u)=$ $\max \{\operatorname{depth}(t), \operatorname{depth}(u)\}$. The weak depth $\operatorname{depth}_{w}(t)$ does not count $\tau$-transitions, meaning that it is defined similar to the depth, except depth ${ }_{w}(\tau t)=\operatorname{depth}_{w}(t)$.

A (closed) substitution, denoted by $\rho, \sigma$, maps variables in $V$ to (closed) terms. Clearly, $t \stackrel{\alpha}{\rightarrow} t^{\prime}$ implies that $\sigma(t) \stackrel{\alpha}{\rightarrow} \sigma\left(t^{\prime}\right)$ for all substitutions $\sigma$. For open terms $t$ and $u$, and a preorder $\sqsubseteq$ (or equivalence $\equiv$ ) on closed terms, we define $t \sqsubseteq u$ (or $t \equiv u$ ) if $\rho(t) \sqsubseteq \rho(u)$ (resp. $\rho(t) \equiv \rho(u)$ ) for all closed substitutions $\rho$.

Summation $\sum\left\{t_{1}, \ldots, t_{n}\right\}$ or $\sum_{i \in\{1, \ldots, n\}} t_{i}$ denotes $t_{1}+\cdots+t_{n}$, where summation over the empty set denotes $\mathbf{0}$. As binding convention, _ $+_{-}$and summation bind weaker than $\alpha_{-}$. For every term $t$ there exists a finite set $\left\{\alpha_{i} t_{i} \mid i \in I\right\}$ of terms and a finite set $Y$ of variables such that $t=\sum_{i \in I} \alpha_{i} t_{i}+\sum_{y \in Y} y$. The $\alpha_{i} t_{i}$ for $i \in I$ and the $y \in Y$ are called 
the summands of $t$. It is easy to see that $t \stackrel{\alpha}{\rightarrow} t^{\prime}$ iff $\alpha t^{\prime}$ is a summand of $t$. The term $\alpha^{n} t$ is obtained from $t$ by prefixing it $n$ times with $\alpha$, i.e., $\alpha^{0} t=t$ and $\alpha^{n+1} t=\alpha\left(\alpha^{n} t\right)$.

A preorder (or equivalence) $R$ is a precongruence (resp. congruence) for $\operatorname{BCCS}(A)$ if $p_{1} R q_{1}$ and $p_{2} R q_{2}$ implies $p_{1}+p_{2} R q_{1}+q_{2}$ and $\alpha p_{1} R \alpha q_{1}$ for all $\alpha \in A_{\tau}$. If a preorder is a precongruence, then clearly the associated equivalence is a congruence. The preorders defined in Def. 2.2, 2.3 and 2.4 are all precongruences for $\operatorname{BCCS}(A)$ G01, G93a, VM01.

Remark 2.5. The requirement that $s_{1} \stackrel{\tau}{\rightarrow}$ implies $s_{2} \stackrel{\tau}{\rightarrow}$ is used to make $\precsim$ WCT, $\precsim$ WF and $\precsim$ WIF a precongruence for $\operatorname{BCCS}(A)$. Without this requirement we would e.g. have $\tau \mathbf{0} \precsim$ WIF $\mathbf{0}$. However, $\tau \mathbf{0}+a \mathbf{0} \swarrow_{W C T} \mathbf{0}+a \mathbf{0}$, because $\varepsilon$ is a completed trace of the first term but not of the second. For $\precsim \mathrm{WF}$ this requirement is in fact needed, in the sense that the version of $\precsim \mathrm{WF}$ with this requirement can be obtained as the congruence-closure for the +-operator of the version from [G93a] without this requirement. A similar observation can be made for $\precsim_{W I F}$. For $\precsim W C T$ the version with the requirement, presented here, appears to be strictly coarser than the BCCS-congruence closure of the version from [G93a] without this requirement. As it is out of the scope of this paper to characterize this congruence closure, which may be most deserving of the name weak completed trace preorder, here we simply employ ${ }_{W C T}$ as defined above.

The requirement that $\mathcal{W} \mathcal{T}\left(s_{1}\right)=\mathcal{W} \mathcal{T}\left(s_{2}\right)$ is needed to make $\precsim$ WIF a precongruence for $\operatorname{BCCS}(A)$. Without this requirement we would e.g. have $\tau a \mathbf{0} \precsim$ WIF $\tau a \mathbf{0}+b \mathbf{0}$. In particular, $(\varepsilon,\{b\})$ is an impossible future not only of the first but also of the second term, because $\tau a \mathbf{0}+b \mathbf{0} \stackrel{\tau}{\rightarrow} a \mathbf{0}$. However, $c(\tau a \mathbf{0})$ L్WIF $c(\tau a \mathbf{0}+b \mathbf{0})$, because $(\varepsilon,\{c b\})$ is an impossible future of the first but not of the second term.

2.4. Axiomatization. An axiomatization is a collection of equations $t \approx u$ or of inequations $t \preccurlyeq u$. The (in)equations in an axiomatization $E$ are referred to as axioms. If $E$ is an equational axiomatization, we write $E \vdash t \approx u$ if the equation $t \approx u$ is derivable from substitution instances of the axioms in $E$ using the rules of equational logic (reflexivity, symmetry, transitivity, and closure under contexts), i.e.,

$$
\overline{t \approx t} \quad \frac{t \approx u}{u \approx t} \quad \frac{t \approx u \quad u \approx v}{t \approx v} \quad \frac{t \approx u}{\alpha t \approx \alpha u} \quad \frac{t_{1} \approx u_{1} t_{2} \approx u_{2}}{t_{1}+t_{2} \approx u_{1}+u_{2}}
$$

For the derivation of an inequation $t \preccurlyeq u$ from an inequational axiomatization $E$, denoted by $E \vdash t \preccurlyeq u$, the rule for symmetry is omitted. We will also allow equations $t \approx u$ in inequational axiomatizations, as an abbreviation of two separate equations $t \preccurlyeq u$ and $u \preccurlyeq t$.

An axiomatization $E$ is sound modulo a preorder $\sqsubseteq$ (or equivalence $\equiv$ ) if for any terms $t, u$, from $E \vdash t \preccurlyeq u$ (or $E \vdash t \approx u$ ) it follows that $\rho(t) \sqsubseteq \rho(u)$ (or $\rho(t) \equiv \rho(u)$ ) for all closed substitutions $\rho$. $E$ is ground-complete for $\sqsubseteq$ (or $\equiv$ ) if for any closed terms $p, q, p \sqsubseteq q$ (or $p \equiv q)$ implies $E \vdash p \preccurlyeq q($ or $E \vdash p \approx q)$. And $E$ is $\omega$-complete if for any terms $t, u$ with $E \vdash \rho(t) \preccurlyeq \rho(u)$ (or $E \vdash \rho(t) \approx \rho(u)$ ) for all closed substitutions $\rho$, we have $E \vdash t \preccurlyeq u$ (or $E \vdash t \approx u$ ). The equational theory of a process algebra modulo a preorder $\sqsubseteq$ (or equivalence $\equiv)$ is said to be finitely based if there exists a finite, $\omega$-complete axiomatization that is sound and ground-complete for the process algebra modulo $\sqsubseteq$ (or $\equiv)$.

The core axioms A1-4 below are sound for $\operatorname{BCCS}(A)$ modulo every semantics in the spectrum depicted in Fig. 1. We assume that A1-4 are included in every axiomatization, 
and write $t=u$ if $\mathrm{A} 1-4 \vdash t \approx u$.

\begin{tabular}{|c|c|c|c|}
\hline A1 & $x+y$ & $\approx$ & $y+x$ \\
\hline A2 & $(x+y)+z$ & $\approx$ & $x+(y+z)$ \\
\hline A3 & $x+x$ & $\approx$ & $x$ \\
\hline$A$ & $x+\mathbf{0}$ & $\approx$ & $x$ \\
\hline
\end{tabular}

\section{Axiomatizability: From Concrete to Weak Semantics}

We present a general method to derive a ground-complete axiomatization for $\operatorname{BCCS}(A)$ modulo a weak semantics from a ground-complete axiomatization for $\operatorname{BCCSP}(A)$ modulo its concrete counterpart. Moreover, if the original axiomatization is $\omega$-complete, then so is the resulting axiomatization.

\subsection{Generating an axiomatization for a corresponding weak semantics.}

Definition 3.1. Given an equivalence $\equiv_{c}$ (resp. preorder $\Xi_{c}$ ) which is a (pre)congruence for $\operatorname{BCCSP}(A)$. A corresponding weak equivalence $\equiv_{w}$ (resp. preorder $\sqsubseteq_{w}$ ) is a (pre)congruence for $\operatorname{BCCS}(A)$ that coincides with $\equiv_{c}$ (resp. $\sqsubseteq_{c}$ ) over closed $\operatorname{BCCSP}(A)$ terms.

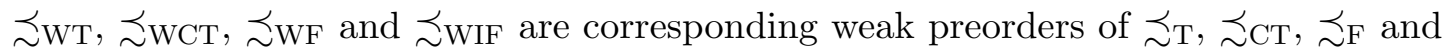
$\precsim_{\mathrm{IF}}$, respectively. Likewise for the four associated equivalences.

Consider an axiomatization that is ground-complete for $\operatorname{BCCSP}(A)$ modulo a concrete (pre)congruence relation. We present an algorithm to generate a ground-complete axiomatization for $\operatorname{BCCS}(A)$ modulo a corresponding weak semantics. The algorithm prescribes the presence of the following two axioms. Actually, an instance of WIF1 is supposed to be present for each $\alpha \in A_{\tau}$.

$$
\begin{aligned}
& \text { WIF1 } \quad \alpha(\tau x+\tau y) \approx \alpha x+\alpha y \\
& \text { WIF2 } \tau x+y \approx \tau x+\tau(x+y)
\end{aligned}
$$

WIF1-2 make it possible to eliminate all non-initial occurrences of $\tau$ within a term (see Prop. 3.8). These two axioms - and hence our algorithm - are sound only for semantics at least as coarse as weak impossible futures semantics. In particular, they are sound for weak failures, completed trace and trace semantics (cf. Fig. 1).

In case of a weak corresponding preorder $\sqsubseteq_{w}$, the algorithm may moreover prescribe the presence of two axioms concerning the introduction or elimination of initial occurrences of $\tau$ 's, which are needed to make the weak preorder under consideration a precongruence (cf. Remark 2.5).

$$
\begin{array}{rrll}
\mathrm{W} 1 & x & \preccurlyeq & \tau x \\
\mathrm{~W} 2 & \tau x & \preccurlyeq & x
\end{array}
$$

W1 must be present if $p \sqsubseteq_{w} q$ for some closed $\operatorname{BCCS}(A)$ terms $p$ and $q$ with $p \stackrel{\tau}{\not \rightarrow}$ and $q \stackrel{\tau}{\rightarrow}$. Likewise, W2 must be present if $p \sqsubseteq_{w} q$ for some closed $\operatorname{BCCS}(A)$ terms $p$ and $q$ with $p \stackrel{\tau}{\rightarrow}$

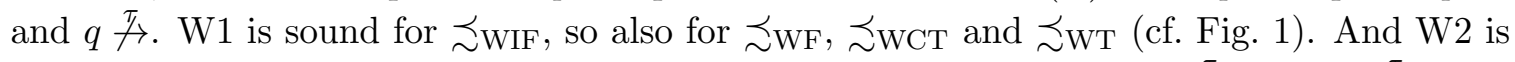
sound for $\precsim_{\mathrm{WT}}$, while for the other three weak preorders $p \sqsubseteq_{w} q$ and $p \stackrel{\tau}{\rightarrow}$ imply $q \stackrel{\tau}{\rightarrow}$.

In case of a weak corresponding equivalence $\equiv_{w}$, the algorithm prescribes the presence of the axiom

$$
\mathrm{WE} \quad x \approx \tau x
$$


if $p \equiv_{w} q$ for some closed $\operatorname{BCCS}(A)$ terms $p$ and $q$ with $p \stackrel{\tau}{\not \rightarrow}$ and $q \stackrel{\tau}{\rightarrow}$. WE is sound for $\equiv_{\mathrm{WT}}$, while $\equiv_{\mathrm{WIF}}, \equiv_{\mathrm{WF}}$ and $\equiv_{\mathrm{WCT}}$ do not require the presence of WE.

Furthermore, the algorithm uses an operator called init- $\tau$ that maps $\operatorname{BCCSP}(A)$ terms to $\operatorname{BCCS}(A)$ terms by renaming initial actions into $\tau$. It is defined inductively by:

$$
\begin{array}{ll}
\operatorname{init}-\tau(\mathbf{0}) & =\mathbf{0} \\
\operatorname{init-\tau }(t+u) & =i n i t-\tau(t)+i n i t-\tau(u) \\
i n i t-\tau(a t) & =\tau t \\
i n i t-\tau(x) & =x
\end{array}
$$

This operator lifts to (in)equations and axiomatizations as expected.

Now we are ready to formulate how an axiomatization $E$ for $\operatorname{BCCSP}(A)$ modulo a concrete semantics is transformed into an axiomatization $\mathcal{A}(E)$ for $\operatorname{BCCS}(A)$ modulo a corresponding weak semantics. First we treat the case of preorders.

Definition 3.2. Let $E$ be an axiomatization for $\operatorname{BCCSP}(A)$ modulo a preorder $\sqsubseteq_{c}$. The axiomatization $\mathcal{A}(E)$ for $\operatorname{BCCS}(A)$ modulo a corresponding weak preorder $\sqsubseteq_{w}$ consists of the following inequations:

(1) E.

(2) $\operatorname{init}-\tau(E)$.

(3) WIF1-2.

(4) If $p \sqsubseteq_{w} q$ for some closed $\operatorname{BCCS}(A)$ terms $p$ and $q$ with $p \stackrel{\pi}{\not \rightarrow}$ and $q \stackrel{\tau}{\rightarrow}$, then $\mathrm{W} 1$ is included.

(5) If $p \sqsubseteq_{w} q$ for some closed $\operatorname{BCCS}(A)$ terms $p$ and $q$ with $p \stackrel{\tau}{\rightarrow}$ and $q \stackrel{\pi}{\rightarrow}$, then W2 is included.

It is essential for the correctness of this approach that axioms do not mix initial and non-initial occurrences of variables. An example of such an (unsafe) inequation is $x \preccurlyeq a x$.

Definition 3.3. A term is said to be safe if no variable has both an initial and a non-initial occurrence in it. An (in)equation $t \preccurlyeq u$ or $t \approx u$ is safe if $t+u$ is safe. An axiomatization is safe if all its axioms are so.

Theorem 3.4. Let $\sqsubseteq_{c}$ be a precongruence for $B C C S P(A)$ and $\sqsubseteq_{w}$ a corresponding weak preorder. Let $E$ be a ground-complete axiomatization for $B C C S P(A)$ modulo $\sqsubseteq_{c}$, which is safe and contains $A 1-4$. Then $\mathcal{A}(E)$ is ground-complete for $B C C S(A)$ modulo $\sqsubseteq_{w}$. If $E$ is moreover $\omega$-complete for $B C C S P(A)$, then $\mathcal{A}(E)$ is $\omega$-complete for $B C C S(A)$.

Note that this theorem does not address the soundness of $\mathcal{A}(E)$ for $\operatorname{BCCS}(A)$ modulo $\sqsubseteq_{w}$. This is left to the user as a separate proof obligation.

The basic ideas behind the method above are as follows. With WIF1-2, each BCCS $(A)$ term can be equated to either a $\operatorname{BCCSP}(A)$ term or a term $\sum_{i \in I} \tau t_{i}$ where the $t_{i}$ are $\operatorname{BCCSP}(A)$ terms (see Prop. 3.8). And with the axioms in init- $\tau(E)$, a derivation of $\sum_{i \in I} a t_{i} \preccurlyeq \sum_{j \in J} a u_{j}$ from $E$ can be converted into a derivation of $\sum_{i \in I} \tau t_{i} \preccurlyeq \sum_{j \in J} \tau u_{j}$ from $\mathcal{A}(E)$. These constitute key steps in the proof of Thm. 3.4 .

The algorithm to generate an axiomatization for $\operatorname{BCCS}(A)$ modulo a weak equivalence from an axiomatization for $\operatorname{BCCSP}(A)$ modulo the corresponding concrete equivalence can be adapted accordingly from the algorithm for preorders.

Definition 3.5. Let $E$ be an axiomatization for $\operatorname{BCCSP}(A)$ modulo an equivalence $\equiv_{c}$. The axiomatization $\mathcal{A}(E)$ for $\operatorname{BCCS}(A)$ modulo a corresponding weak equivalence $\equiv_{w}$ consists of the following equations: 
(1) $E$.

(2) $\operatorname{init}-\tau(E)$.

(3) WIF1-2.

(4) If $p \equiv_{w} q$ for some closed $\operatorname{BCCS}(A)$ terms $p$ and $q$ with $p \stackrel{\tau}{\rightarrow}$ and $q \not{\pi} \rightarrow$, then WE is included.

Theorem 3.6. Let $\equiv_{c}$ be a congruence for $B C \operatorname{CSP}(A)$ and $\equiv_{w}$ a corresponding weak equivalence. Let $E$ be a ground-complete axiomatization for $\operatorname{BCCSP}(A)$ modulo $\equiv_{c}$, which is safe and contains A1-4. Then $\mathcal{A}(E)$ is ground-complete for BCCS $(A)$ modulo $\equiv_{w}$. If $E$ is moreover $\omega$-complete for $B C C S P(A)$, then $\mathcal{A}(E)$ is $\omega$-complete for $B C C S(A)$.

3.2. Correctness of the transformations. We establish the correctness of the algorithms for preorders and equivalences. We will only prove Thm. 3.4 for preorders, as Thm. 3.6 for equivalences can be proved following the same lines. For a start, we show that the following equations can be derived from A1-4+WIF1-2:

$$
\begin{aligned}
\text { D1 } \quad \tau(\tau x+y) & \approx \tau x+y \\
\text { D2 } \quad \alpha\left(\sum_{i \in I} \tau x_{i}+y\right) & \approx \sum_{i \in I} \alpha x_{i}+\alpha\left(\sum_{i \in I} x_{i}+y\right)
\end{aligned}
$$

Lemma 3.7. D1-2 are derivable from A1-4+WIF1-2.

Proof. For D1,

$$
\begin{array}{rlr}
\tau(\tau x+y) & \approx \tau(\tau x+\tau(x+y)) & (\mathrm{WIF} 2) \\
& \approx \tau x+\tau(x+y) & (\mathrm{WIF} 1) \\
& \approx \tau x+y & (\mathrm{WIF} 2)
\end{array}
$$

For D2, we apply induction on $|I|$. The base case $I=\emptyset$ is trivial. For $|I| \geq 1$, pick an $i_{0} \in I$.

$$
\begin{aligned}
\alpha\left(\sum_{i \in I} \tau x_{i}+y\right) & =\alpha\left(\tau x_{i_{0}}+\sum_{i \in I \backslash\left\{i_{0}\right\}} \tau x_{i}+y\right) \\
& \approx \alpha\left(\tau x_{i_{0}}+\tau\left(x_{i_{0}}+\sum_{i \in I \backslash\left\{i_{0}\right\}} \tau x_{i}+y\right)\right) \\
& \approx \alpha x_{i_{0}}+\alpha\left(x_{i_{0}}+\sum_{i \in I \backslash\left\{i_{0}\right\}} \tau x_{i}+y\right) \\
& \approx \alpha x_{i_{0}}+\sum_{i \in I \backslash\left\{i_{0}\right\}} \alpha x_{i}+\alpha\left(x_{i_{0}}+\sum_{i \in I \backslash\left\{i_{0}\right\}} x_{i}+y\right) \quad \text { (WIF2) } \\
& =\sum_{i \in I} \alpha x_{i}+\alpha\left(\sum_{i \in I} x_{i}+y\right)
\end{aligned}
$$

The following proposition on the elimination of $\tau$ 's from $\operatorname{BCCS}(A)$ terms will play a key role in the proof of Thm. 3.4 .

Proposition 3.8. Let $t$ be a $\operatorname{BCCS}(A)$ term.

(1) If $t \not{t} \rightarrow$, then A1-4+WIF1-2 $\vdash t \approx t^{\prime}$ for some $\operatorname{BCCSP}(A)$ term $t^{\prime}$.

(2) If $t \stackrel{\tau}{\rightarrow}$, then A1-4+WIF1-2 $\vdash t \approx \sum_{i \in I} \tau t_{i}$ where $I \neq \emptyset$ and the $t_{i}$ are $\operatorname{BCCSP}(A)$ terms.

Proof. It is easy to see that A1-4 and WIF1-2 equate only terms of equal weak depth. For convenience, "A1-4+WIF1-2 $\vdash$ " is omitted here. 
(1) We apply induction on the weak depth of $t$. Since $t \stackrel{\pi}{\rightarrow}, t=\sum_{i \in I} a_{i} t_{i}+\sum_{j \in J} x_{j}$. For each $i \in I$,

$$
t_{i} \approx \sum_{k \in K_{i}} \tau t_{k}^{\prime}+\sum_{\ell \in L_{i}} b_{\ell} t_{\ell}^{\prime}+\sum_{m \in M_{i}} y_{m}
$$

Moreover, by means of D1 we can guarantee that for each $k \in K_{i}, t_{k}^{\prime} \frac{\pi}{7}$. So, by D2,

$$
a_{i} t_{i} \approx \sum_{k \in K_{i}} a_{i} t_{k}^{\prime}+a_{i}\left(\sum_{k \in K_{i}} t_{k}^{\prime}+\sum_{\ell \in L_{i}} b_{\ell} t_{\ell}^{\prime}+\sum_{m \in M_{i}} y_{m}\right)
$$

For each $k \in K_{i}$, by induction, $t_{k}^{\prime} \approx t_{k}^{\prime \prime}$ where $t_{k}^{\prime \prime}$ is a $\operatorname{BCCSP}(A)$ term. Likewise, by induction, $\sum_{k \in K_{i}} t_{k}^{\prime}+\sum_{\ell \in L_{i}} b_{\ell} t_{\ell}^{\prime}+\sum_{m \in M_{i}} y_{m} \approx t_{i}^{\prime \prime \prime}$ where $t_{i}^{\prime \prime \prime}$ is a $\operatorname{BCCSP}(A)$ term. Hence,

$$
t=\sum_{i \in I} a_{i} t_{i}+\sum_{j \in J} x_{j} \approx \sum_{i \in I}\left(\sum_{k \in K_{i}} a_{i} t_{k}^{\prime \prime}+a_{i} t_{i}^{\prime \prime \prime}\right)+\sum_{j \in J} x_{j}
$$

And this last term is in $\operatorname{BCCSP}(A)$.

(2) Since $t \stackrel{\tau}{\rightarrow}, t \approx \sum_{i \in I} \tau t_{i}+\sum_{j \in J} a_{j} t_{j}+\sum_{k \in K} x_{k}$ where $I \neq \emptyset$. Moreover, by means of D1 we can guarantee that for each $i \in I, t_{i} \not$ t . Pick an $i_{0} \in I$. By WIF2,

$$
t \approx \sum_{i \in I} \tau t_{i}+\tau\left(t_{i_{0}}+\sum_{j \in J} a_{j} t_{j}+\sum_{k \in K} x_{k}\right)
$$

And by (1), the terms $t_{i}$ and $a_{j} t_{j}$ can all be equated to $\operatorname{BCCSP}(A)$ terms.

We proceed to prove that a derivation of $t \preccurlyeq u$ from $E$ yields a derivation of $\operatorname{init}-\tau(t \preccurlyeq u)$ from init- $\tau(E)$. First we establish a lemma as a stepping stone toward this result.

Lemma 3.9. Let $\sigma(t)$ be a $\operatorname{BCCSP}(A)$ term, and let $t$ be safe. Then

$$
\text { init- } \tau(\sigma(t))=\sigma^{\prime}(\text { init- } \tau(t))
$$

where $\sigma^{\prime}(x)=$ init- $\tau(\sigma(x))$ if $x$ has an initial occurrence in $t$ and $\sigma^{\prime}(x)=\sigma(x)$ otherwise.

Proof. By induction on the structure of $t$.

- $t=\mathbf{0}$ : We have init- $\tau(\sigma(\mathbf{0}))=\mathbf{0}=\sigma^{\prime}($ init- $\tau(\mathbf{0}))$.

- $t=x$ : We have init- $\tau(\sigma(x))=\sigma^{\prime}(x)=\sigma^{\prime}($ init- $\tau(x))$.

- $t=t_{1}+t_{2}$ : For $n=1,2$ we define $\sigma_{n}(x)=i n i t-\tau(\sigma(x))$ if $x$ has an initial occurrence in $t_{n}$ and $\sigma_{n}(x)=\sigma(x)$ otherwise. Since $t_{1}+t_{2}$ is safe, $\sigma^{\prime}$ and $\sigma_{n}$ coincide over $\operatorname{var}\left(t_{n}\right)$ for both $n=1$ and $n=2$. Hence,

$$
\begin{aligned}
\operatorname{init-\tau }\left(\sigma\left(t_{1}+t_{2}\right)\right) & =\operatorname{init}-\tau\left(\sigma\left(t_{1}\right)\right)+i n i t-\tau\left(\sigma\left(t_{2}\right)\right) \\
& =\sigma_{1}\left(\text { init- } \tau\left(t_{1}\right)\right)+\sigma_{2}\left(\text { init- } \tau\left(t_{2}\right)\right) \quad \text { (by induction) } \\
& =\sigma^{\prime}\left(\text { init- } \tau\left(t_{1}\right)\right)+\sigma^{\prime}\left(\text { init- } \tau\left(t_{2}\right)\right) \\
& =\sigma^{\prime}\left(\operatorname{init}-\tau\left(t_{1}+t_{2}\right)\right)
\end{aligned}
$$

- $t=a t^{\prime}$ : Since $\sigma$ and $\sigma^{\prime}$ coincide over $\operatorname{var}\left(t^{\prime}\right)$,

$$
\text { init- } \tau\left(\sigma\left(a t^{\prime}\right)\right)=\operatorname{init}-\tau\left(a \sigma\left(t^{\prime}\right)\right)=\tau \sigma\left(t^{\prime}\right)=\tau \sigma^{\prime}\left(t^{\prime}\right)=\sigma^{\prime}\left(\tau t^{\prime}\right)=\sigma^{\prime}\left(\operatorname{init}-\tau\left(a t^{\prime}\right)\right)
$$


Proposition 3.10. Let $E$ be a safe axiomatization for $\operatorname{BCCSP}(A)$ and suppose that $E \vdash t \preccurlyeq u$. Then

$$
\operatorname{init}-\tau(E) \vdash \operatorname{init}-\tau(t \preccurlyeq u)
$$

Proof. By induction on the derivation of $t \preccurlyeq u$ from $E$. The cases of reflexivity, transitivity and closure under context are straightforward. We focus on the case of a substitution instance of an axiom, meaning that there are an axiom $v \preccurlyeq w$ in $E$ and a substitution $\sigma$ such that $\sigma(v)=t$ and $\sigma(w)=u$. Since $v$ and $w$ are safe, Lem. 3.9 can be applied to both $v$ and $w$. We define $\sigma^{\prime}(x)=$ init- $\tau(\sigma(x))$ if $x$ has an initial occurrence in $v+w$ and $\sigma^{\prime}(x)=\sigma(x)$ otherwise. Since $v \preccurlyeq w$ is safe, $\sigma^{\prime}$ can be used in the application of Lem. 3.9 to both $v$ and $w$. By two applications of Lem. 3.9 and one of the axiom init- $\tau(v \preccurlyeq w)$ in init- $\tau(E)$ we derive

$$
\operatorname{init}-\tau(t)=\operatorname{init}-\tau(\sigma(v))=\sigma^{\prime}(\operatorname{init}-\tau(v)) \preccurlyeq \sigma^{\prime}(\operatorname{init}-\tau(w))=\operatorname{init}-\tau(\sigma(w))=\operatorname{init}-\tau(u)
$$

Now we are ready to prove Thm. 3.4 for preorders. As said before, the case of equivalences can be proved following the same lines.

Proof. Let $E$ be a ground-complete axiomatization for $\operatorname{BCCSP}(A)$ modulo $\sqsubseteq_{c}$. Suppose that $t \sqsubseteq_{w} u$ where either the $\operatorname{BCCS}(A)$ terms $t$ and $u$ are closed or $E$ is $\omega$-complete for $\operatorname{BCCSP}(A)$. To show that $\mathcal{A}(E) \vdash t \preccurlyeq u$, we distinguish four cases. Note, with regard to Prop. 3.8, that A1-4 and WIF1-2 equate closed terms only with closed terms.

(1) $t \underset{7}{\not}$ and $u \not / 7$. By Prop. 3.8(1), from A1-4+WIF1-2 we can derive $t \approx t^{\prime}$ and $u \approx u^{\prime}$ where $t^{\prime}$ and $u^{\prime}$ are $\operatorname{BCCSP}(A)$ terms (and closed if $t$ and $u$ are closed). Since $t \sqsubseteq_{w} u$ and A1-4+WIF1-2 are sound for $\operatorname{BCCS}(A)$ modulo $\equiv_{w}$, we have $t^{\prime} \sqsubseteq_{w} u^{\prime}$. Since $t^{\prime}$ and $u^{\prime}$ are $\operatorname{BCCSP}(A)$ terms and $\sqsubseteq_{w}$ coincides with $\sqsubseteq_{c}$ over closed $\operatorname{BCCSP}(A)$ terms, $t^{\prime} \sqsubseteq_{c} u^{\prime}$. Since $E$ is ground-complete for $\operatorname{BCCSP}(A)$ modulo $\sqsubseteq_{c}$, and $\omega$-complete if $t$ or $u$ is not closed, it follows that $E \vdash t^{\prime} \preccurlyeq u^{\prime}$. Hence $\mathcal{A}(E) \vdash t \preccurlyeq u$.

(2) $t \stackrel{\tau}{\rightarrow}$ and $u \stackrel{\tau}{\rightarrow}$. By Prop. $3.8(2)$, from A1-4+WIF1-2 we can derive $t \approx \sum_{i \in I} \tau t_{i}$ and $u \approx \sum_{j \in J} \tau u_{j}$ where $I, J \neq \emptyset$ and the $t_{i}$ and $u_{j}$ are $\operatorname{BCCSP}(A)$ terms (and closed if $t$ and $u$ are closed). Since $t \sqsubseteq_{w} u$ and A1-4+WIF1-2 are sound for $\operatorname{BCCS}(A)$ modulo $\equiv_{w}$, we have $\sum_{i \in I} \tau t_{i} \sqsubseteq_{w} \sum_{j \in J} \tau u_{j}$. Pick an $a \in A$. We have $\sum_{i \in I} a t_{i} \equiv_{\mathrm{WIF}} a\left(\sum_{i \in I} \tau t_{i}\right) \sqsubseteq_{w}$ $a\left(\sum_{j \in J} \tau u_{j}\right) \equiv_{\mathrm{WIF}} \sum_{j \in J} a u_{j}$. So $\sum_{i \in I} a t_{i} \sqsubseteq_{w} \sum_{j \in J} a u_{j}$. Since the $t_{i}$ and $u_{j}$ are $\operatorname{BCCSP}(A)$ terms and $\sqsubseteq_{w}$ coincides with $\sqsubseteq_{c}$ over closed $\operatorname{BCCSP}(A)$ terms, $\sum_{i \in I} a t_{i} \sqsubseteq_{c}$ $\sum_{j \in J} a u_{j}$. Since $E$ is ground-complete for $\operatorname{BCCSP}(A)$ modulo $\sqsubseteq_{c}$, and $\omega$-complete if $t$ or $u$ is not closed, it follows that $E \vdash \sum_{i \in I} a t_{i} \preccurlyeq \sum_{j \in J} a u_{j}$. So, since $E$ is safe, by Prop. 3.10, $\mathcal{A}(E) \vdash \sum_{i \in I} \tau t_{i}=i n i t-\tau\left(\sum_{i \in I} a t_{i}\right) \preccurlyeq i n i t-\tau\left(\sum_{j \in J} a u_{j}\right)=\sum_{j \in J} \tau u_{j}$. Hence $\mathcal{A}(E) \vdash t \preccurlyeq u$.

(3) $t \stackrel{\tau}{\rightarrow}$ and $u \stackrel{\tau}{\rightarrow}$. By requirement (4) of Def. 3.2. W1 is included in $\mathcal{A}(E)$. We have $\mathcal{A}(E) \vdash t \preccurlyeq \tau t \preccurlyeq \tau u \approx u$. The first step follows from W1, the second from case (2), and the third from D1 together with $u \stackrel{\tau}{\rightarrow}$.

(4) $t \stackrel{\tau}{\rightarrow}$ and $u \stackrel{\tau}{7 \rightarrow}$. By requirement (5) of Def. 3.2. W2 is included in $\mathcal{A}(E)$. We have $\mathcal{A}(E) \vdash t \approx \tau t \preccurlyeq \tau u \preccurlyeq u$. The first step follows from D1 together with $t \stackrel{\tau}{\rightarrow}$, the second from case (2), and the third from W2.

Remark 3.11. There is an alternative approach for the method introduced in this section that avoids the use of the init- $\tau$ operator. That is, clause (2) in the construction of $\mathcal{A}(E)$ in Def. 3.2 is omitted. Moreover, the axiomatization $E$ does not have to be safe. And while we chose to ignore preservation of soundness, as the init- $\tau$ operator would give rise to some 
technical complications, in the alternative approach, soundness of $E$ modulo the strong semantics yields soundness of $\mathcal{A}(E)$ modulo the weak semantics in a straightforward fashion, provided we assume the strong preorder (or equivalence) is included in the corresponding weak one. However, the price to pay is that this alternative method only works for groundcompleteness (so not for $\omega$-completeness), and assumes the so-called Fresh Atom Principle (see e.g. G05]). We briefly sketch the idea behind this alternative approach.

The axiomatization $E$ is required to be sound and ground-complete for $\operatorname{BCCSP}(A)$. The crucial case (2) in the proof of Thm. 3.4 can now be tackled without init- $\tau$. As before we arrive at $\sum_{i \in I} \tau t_{i} \sqsubseteq w \sum_{j \in J} \tau u_{j}$, but as we are dealing with ground-completeness only, the $t_{i}$ and $u_{j}$ are closed terms. Let $a$ be a fresh action which is not in the alphabet $A$. The last mentioned relation together with the soundness of WIF1 yields $\sum_{i \in I} a t_{i} \sqsubseteq_{w} \sum_{j \in J} a u_{j}$. This implies $\sum_{i \in I} a t_{i} \sqsubseteq_{c} \sum_{j \in J} a u_{j}$. Since $a$ is fresh, renaming it into $\tau$ yields $\sum_{i \in I} \tau t_{i} \sqsubseteq_{c} \sum_{j \in J} \tau u_{j}$, where $\tau$ is interpreted as a concrete action. So by ground-completeness, $E \vdash \sum_{i \in I} \tau t_{i} \preccurlyeq$ $\sum_{j \in J} \tau u_{j}$, which implies $\mathcal{A}(E) \vdash t \preccurlyeq u$.

To see why this reasoning does not extend to $\omega$-completeness, assume that the $t_{i}$ and $u_{j}$ are open terms. Then the inequation $\sum_{i \in I} \tau t_{i} \sqsubseteq w \sum_{j \in J} \tau u_{j}$ really means that $\sum_{i \in I} \tau \sigma\left(t_{i}\right) \sqsubseteq w \sum_{j \in J} \tau \sigma\left(u_{j}\right)$ for any closed substitution $\sigma$ in $\operatorname{BCCS}(A)$. From that, one may not conclude that this equation also holds for any closed substitution $\sigma$ in $\operatorname{BCCS}(A \cup\{a\})$, and the latter is needed to infer $\sum_{i \in I} a t_{i} \sqsubseteq_{c} \sum_{j \in J} a u_{j}$.

\section{Application to Failures, Completed Traces and Traces}

In this section, the algorithm from the previous section is applied to produce axiomatizations for $\operatorname{BCCS}(A)$ modulo the weak failures, completed trace and trace preorders and equivalences from axiomatizations for $\operatorname{BCCSP}(A)$ modulo their concrete counterparts.

4.1. Failures semantics. According to [CFLN08, A1-4 together with the axiom

$$
\text { F1 } \quad a(x+y) \preccurlyeq a x+a(y+z)
$$

constitute a sound and ground-complete axiomatization for $\operatorname{BCCSP}(A)$ modulo $\precsim_{F}$. If $|A|=\infty$ then this axiomatization is $\omega$-complete, while if $|A|<\infty$ then a finite basis for the inequational theory of $\operatorname{BCCSP}(A)$ modulo $\precsim_{\mathrm{F}}$ is obtained by adding the following axiom:

$$
\text { F2 } \quad \sum_{a \in A} a x_{a} \preccurlyeq \sum_{a \in A} a x_{a}+y .
$$

Our algorithm from the previous section produces a ground-complete axiomatization for $\operatorname{BCCS}(A)$ modulo $\precsim \mathrm{WF}$, which consists of A1-4, WIF1-2 and W1 together with

$$
\mathrm{F}^{\prime} \quad \alpha(x+y) \preccurlyeq \alpha x+\alpha(y+z)
$$

It is not hard to see that this axiomatization is sound modulo $\precsim$ WF. F1 is extended to F1' (allowing initial $\tau$ 's) in light of clause (2), WIF1-2 are included in light of clause (3), and W1 in light of clause (4) of Def. 3.2 .

The axiomatization can be simplified: the following axioms together with A1-4 and WIF1 suffice.

$$
\begin{array}{lrll}
\mathrm{WIF}^{\prime} & \tau(x+y) & \preccurlyeq \tau x+y \\
\mathrm{~W}^{\prime} & x & \preccurlyeq \tau x+y
\end{array}
$$


On the one hand, WIF2 ${ }^{\prime}$ and $\mathrm{W}^{\prime}$ are clearly sound modulo $\precsim W F$. On the other hand, W1 follows directly from $\mathrm{W}^{\prime}$ (taking $y=\mathbf{0}$ ) and A4. Furthermore, the two directions of WIF2 can be derived as follows: by WIF $2^{\prime}$ and A3, $\tau(x+y)+\tau x \preccurlyeq \tau x+y$; and by WIF2' (with $y=x$ ) and $\mathrm{W} 1, \tau x+y \preccurlyeq \tau x+x+y \preccurlyeq \tau x+\tau(x+y)$. Finally, $\mathrm{F} 1^{\prime}$ can be derived as follows: by $\mathrm{W} 1^{\prime}$, $x+y \preccurlyeq \tau x+y+z \preccurlyeq \tau x+\tau(y+z)$; so by WIF1, $\alpha(x+y) \preccurlyeq \alpha(\tau x+\tau(y+z)) \approx \alpha x+\alpha(y+z)$.

According to Thm. 3.4, if $|A|=\infty$, then this axiomatization is $\omega$-complete. And if $1<|A|<\infty$, then $\mathrm{F} 2$ and init- $\tau(\mathrm{F} 2)$ have to be added to make the axiomatization $\omega$-complete. But the latter inequation, $\sum_{a \in A} \tau x_{a} \preccurlyeq \sum_{a \in A} \tau x_{a}+y$, can be derived using $\mathrm{W} 1^{\prime}$ and WIF1.

Theorem 4.1. A1-4+WIF1+WIF2'+W1' is sound and ground-complete for BCCS $(A)$ modulo $\precsim \mathrm{WF}$. If $|A|=\infty$, then it is also $\omega$-complete. If $|A|<\infty$, then the axiomatization becomes $\omega$-complete by adding the (sound) axiom $\mathrm{F} 2$.

According to [G01], A1-4 together with the axioms

FE1 $\quad a x+a(y+z) \approx a x+a(x+y)+a(y+z)$

$\mathrm{FE}^{*} \quad a(b x+u)+a(b y+v) \approx a(b x+b y+u)+a(b y+v)$

constitute a sound and ground-complete axiomatization for $\operatorname{BCCSP}(A)$ modulo $\simeq_{\mathrm{F}}$. As remarked in [FN05], in the presence of FE1, axiom FE2* can be simplified to

$$
\text { FE2 } a(x+b y)+a(x+b y+b z) \approx a(x+b x+b z) .
$$

Moreover, in [FN05] it was proved that if $|A|=\infty$ then this axiomatization is $\omega$-complete, while if $|A|<\infty$ then a finite basis for the inequational theory of $\operatorname{BCCSP}(A)$ modulo $\precsim_{\mathrm{F}}$ is obtained by adding the following axiom:

FE3 $a\left(x+\sum_{b \in A} b z_{b}\right)+a\left(x+y+\sum_{b \in A} b z_{b}\right) \approx a\left(x+y+\sum_{b \in A} b z_{b}\right)$.

Our algorithm from the previous section produces a ground-complete axiomatization for $\operatorname{BCCS}(A)$ modulo $\simeq$ WF, which consists of A1-4 and WIF1-2 together with

$$
\begin{aligned}
& \text { FE1 } \quad \alpha x+\alpha(y+z) \approx \alpha x+\alpha(x+y)+\alpha(y+z) \\
& \text { FE2 } \quad \alpha(x+b y)+\alpha(x+b y+b z) \approx \alpha(x+b x+b z) .
\end{aligned}
$$

It is not hard to see that this axiomatization is sound modulo $\simeq_{\mathrm{WF}}$. FE1-2 are extended (allowing initial $\tau$ 's) in light of clause (2) of Def. 3.5 and WIF1-2 are included in light of clause (3).

Also this axiomatization can be simplified: the following axiom together with A1-4 and WIF1-2 suffices.

$$
\mathrm{WFE} \quad a x+\tau(a y+z) \approx \tau(a x+a y+z)
$$

On the one hand, WFE is a direct consequence of WIF2 and FE2': $a x+\tau(a y+z)=$ $\tau(a y+z)+\tau(a x+a y+z)=\tau(a x+a y+z)$. On the other hand, the instances of FE1' and FE2 ${ }^{\prime}$ with $\alpha=\tau$ can be derived with two applications of WIF2 and with WIF2 and WFE, respectively:

$$
\begin{aligned}
& \tau x+\tau(x+y)+\tau(y+z) \approx \tau x+y+\tau(y+z) \approx \tau x+\tau(y+z)+\tau(y+y+z) \approx \tau x+\tau(y+z) \\
& \tau(x+b y)+\tau(x+b y+b z) \approx \tau(x+b y)+b z \approx \tau(x+b x+b z) .
\end{aligned}
$$

The general instances of FE1' and FE2' now follow with WIF1.

In G97] it was already remarked that A1-4+WIF1-2+WFE is a sound and groundcomplete axiomatization for $\operatorname{BCCS}(A)$ modulo $\simeq$ WF. 
According to Thm. 3.6, if $|A|=\infty$, then this axiomatization is $\omega$-complete. And if $1<|A|<\infty$, then FE3 and init- $\tau$ (FE3) have to be added to make the axiomatization $\omega$-complete. But from the latter inequation,

$$
\mathrm{FE}^{\prime} \quad \tau\left(x+\sum_{b \in A} b z_{b}\right)+\tau\left(x+y+\sum_{b \in A} b z_{b}\right) \approx \tau\left(x+y+\sum_{b \in A} b z_{b}\right)
$$

FE3 can be derived using WIF1.

Theorem 4.2. A1-4+WIF1-2+WFE is sound and ground-complete for BCCS(A) modulo $\simeq_{\mathrm{WF}}$. If $|A|=\infty$, then it is also $\omega$-complete. If $|A|<\infty$, then the axiomatization becomes $\omega$-complete by adding the (sound) axiom FE3'.

In AFI07, FGP07, an algorithm is presented which takes as input a sound and groundcomplete inequational axiomatization for BCCSP modulo a preorder $\sqsubseteq$ which includes the ready simulation preorder and is initials preserving 5 and generates as output an equational axiomatization which is sound and ground-complete for BCCSP modulo the corresponding equivalence-its kernel: $\sqsubseteq \cap \sqsubseteq^{-1}$. Moreover, if the original axiomatization is $\omega$-complete, then so is the resulting axiomatization. Using this algorithm, the above-mentioned axiomatization of $\simeq_{F}$ could have been obtained from the one of $\precsim_{F}$.

In CFG08 we lifted this result to weak semantics, which makes the aforementioned algorithm applicable to all 87 preorders surveyed in G93a that are at least as coarse as the ready simulation preorder. In [CFG09] we obtain an alternative proof of Thm. 4.2 by applying the algorithm of [CFG08 to the axiomatizations of Thm. 4.1.

4.2. Completed trace semantics. According to G01, A1-4 together with the axiom

$$
\mathrm{CTE} \quad a(b w+c x+y+z) \approx a(b w+y)+a(c x+z)
$$

constitute a sound and ground-complete axiomatization for $\operatorname{BCCSP}(A)$ modulo $\simeq_{\mathrm{CT}}$. After adding the axiom FE1, the axiomatization becomes $\omega$-complete G90.

By applying our algorithm, we obtain a ground-complete axiomatization for $\operatorname{BCCS}(A)$ modulo $\simeq_{\mathrm{WCT}}$, which consists of A1-4, WIF1-2 and

$$
\mathrm{CTE}^{\prime} \quad \alpha(b w+c x+y+z) \approx \alpha(b w+y)+\alpha(c x+z)
$$

It is not hard to see that this axiomatization is sound modulo $\simeq_{\mathrm{WCT}}$. CTE is extended (allowing initial $\tau$ 's) in light of clause (2) of Def. 3.2. This axiomatization is also $\omega$-complete, since FE1' can be derived, as shown above. Moreover, CTE' follows from CTE and WIF1.

Theorem 4.3. A1-4+WIF1-2+CTE is sound, ground-complete as well as $\omega$-complete for $B C C S(A)$ modulo $\simeq \mathrm{WCT}$.

The axiomatization A1-4 together with

$$
\begin{aligned}
& \text { CT1 } \quad a x \preccurlyeq a x+y \\
& \mathrm{CT} 2 \quad a(b w+c x+y+z) \preccurlyeq a(b w+y)+a(c x+z)
\end{aligned}
$$

from [G01] is sound and ground-complete for $\operatorname{BCCSP}(A)$ modulo $\precsim$ CT. After adding the axiom $\mathrm{F} 1$, the axiomatization becomes $\omega$-complete. This $\omega$-completeness result follows from the $\omega$-completeness of the above axiomatization for $\operatorname{BCCSP}(A)$ modulo $\simeq_{\mathrm{CT}}$. Namely, suppose that $t \precsim_{\mathrm{CT}} u$. If $t$ does not contain a summand of the form $a t^{\prime}$, then clearly $t$ and $u$ must consist of exactly the same variable summands, so that $t=u$. And if $t$ contains a

\footnotetext{
${ }^{5}$ Initials preserving means that $p \sqsubseteq q$ implies $\mathcal{I}(p) \subseteq \mathcal{I}(q)$.
} 
summand $a t^{\prime}$, then by CT1, $t \preccurlyeq t+u$. Since $t+u \simeq_{\mathrm{CT}} u$, derivability of $t+u \approx u$ from A1-4, CT1-2, F1 follows from the aforementioned $\omega$-completeness result for $\operatorname{BCCSP}(A)$ modulo $\simeq_{\mathrm{CT}}$, using that $\mathrm{CTE}$ follows from CT1-2, and FE1 from CT1 and F1.

By applying the algorithm, we obtain a ground-complete axiomatization for $\operatorname{BCCS}(A)$

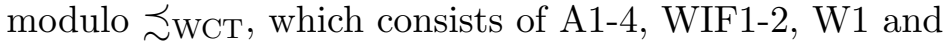

$$
\begin{aligned}
& \mathrm{CT}^{\prime} \quad \alpha x \preccurlyeq \alpha x+y \\
& \mathrm{CT}^{\prime} \quad \alpha(b w+c x+y+z) \preccurlyeq \alpha(b w+y)+\alpha(c x+z)
\end{aligned}
$$

It is not hard to see that this axiomatization is sound modulo $\precsim$ WCT. CT1-2 are extended (allowing initial $\tau$ 's) in light of clause (2) of Def. 3.2 .

WIF1+WIF2' $+\mathrm{W}^{\prime}+\mathrm{CT} 1^{\prime}$ together with A1-4 suffice, because $\mathrm{CT} 2^{\prime}$ can be derived using W1 and WIF1: $\alpha(b w+c x+y+z) \preccurlyeq \alpha(\tau(b w+y)+\tau(c x+z)) \approx \alpha(b w+y)+\alpha(c x+z)$.

We conclude that $\mathrm{A} 1-4+\mathrm{WIF} 1+\mathrm{WIF} 2^{\prime}+\mathrm{W} 1^{\prime}+\mathrm{CT}^{\prime}$ is ground-complete for $\operatorname{BCCS}(A)$ modulo $\precsim$ WCT. It is also $\omega$-complete, since $\mathrm{F} 1^{\prime}$ can be derived, as shown before.

Theorem 4.4. A1-4+WIF1+WIF2' $+\mathrm{W} 1^{\prime}+\mathrm{CT}^{\prime}{ }^{\prime}$ is sound, ground-complete and $\omega$-complete for $B C C S(A)$ modulo $\precsim \mathrm{WCT}$.

4.3. Trace semantics. According to G01, G90], A1-4 together with the axiom

$$
\mathrm{TE} \quad a x+a y \approx a(x+y)
$$

constitute a sound and ground-complete axiomatization for $\operatorname{BCCSP}(A)$ modulo $\simeq_{\mathrm{T}}$. If $|A|>1$ it is even $\omega$-complete [G90].

By applying our algorithm, we obtain a ground-complete axiomatization for $\operatorname{BCCS}(A)$ modulo $\simeq_{\mathrm{CT}}$, consisting of A1-4, WIF1-2, WE and

$$
\mathrm{TE}^{\prime} \quad \alpha x+\alpha y \approx \alpha(x+y)
$$

If $|A|>1$ it is even $\omega$-complete. It is easy to see that it is sound modulo $\simeq_{\mathrm{CT}}$. Clearly, owing to WE, WIF1-2 are redundant.

Theorem 4.5. A1-4+WE+TE is sound and ground-complete for BCCS(A) modulo $\simeq_{\mathrm{CT}}$. If $|A|>1$, then this axiomatization is also $\omega$-complete.

The axiomatization A1-4 together with

$$
\begin{aligned}
& \mathrm{T} 1 \quad a(x+y) \preccurlyeq a x+a y \\
& \text { T2 } \quad x \preccurlyeq x+y
\end{aligned}
$$

from [G01] is sound and ground-complete for $\operatorname{BCCSP}(A)$ modulo $\precsim_{\mathrm{T}}$. If $|A|>1$, then it is also $\omega$-complete. This $\omega$-completeness result follows from the $\omega$-completeness of the above axiomatization for $\operatorname{BCCSP}(A)$ modulo $\simeq_{\mathrm{T}}$. Namely, suppose that $t \precsim_{\mathrm{T}} u$. Then by $\mathrm{T} 2$, $t \preccurlyeq t+u$. Since $t+u \simeq_{\mathrm{T}} u$, derivability of $t+u \approx u$ from A1-4, T1-2 follows from the aforementioned $\omega$-completeness result for $\operatorname{BCCSP}(A)$ modulo $\simeq_{\mathrm{T}}$, using that $\mathrm{TE}$ follows from T1-2.

By applying the algorithm, we obtain a ground-complete axiomatization for $\operatorname{BCCS}(A)$

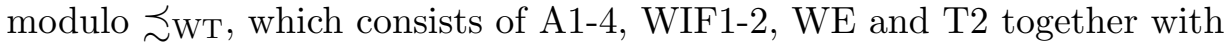

$$
\mathrm{T} 1^{\prime} \quad \alpha(x+y) \preccurlyeq \alpha x+\alpha y
$$

It is not hard to see that this axiomatization is sound modulo $\precsim \mathrm{WT}$. T1 is extended in light of clause (2) and WE is introduced in light of clauses (4) and (5) of Def. 3.2. Again, owing to WE, WIF1-2 are redundant. 
Theorem 4.6. A1-4+T $1^{\prime}+\mathrm{T} 2+\mathrm{WE}$ is sound and ground-complete for BCCS(A) modulo $\precsim \mathrm{WT}$. If $|A|>1$, then this axiomatization is also $\omega$-complete.

If $|A|=1$, then $x \preccurlyeq a x$ needs to be added to make the axiomatization $\omega$-complete (cf. G90]). This is the only corner case where our approach breaks down, due to the fact that this axiom is not safe: the variable $x$ has both an initial and a non-initial occurrence.

Admittedly, the application of Defs. 3.5 and 3.2 to trace semantics is not so interesting, because the axiom WE allows to eliminate all $\tau$ 's from terms, so that the weak setting trivially reduces to the concrete setting.

\section{Ground-Completeness for Impossible Futures}

This section concerns the axiomatizability of impossible futures semantics.

5.1. Concrete impossible futures preorder. We prove that A1-4 together with the axioms

$$
\begin{aligned}
& \text { IF1 } \quad a(x+y) \preccurlyeq a x+a y \\
& \text { IF2 } a x+a(y+z) \approx a(x+y)+a x+a(y+z)
\end{aligned}
$$

constitute a sound and ground-complete axiomatization for $\operatorname{BCCSP}(A)$ modulo $\precsim$ IF .

Theorem 5.1. A1-4+IF1-2 is sound and ground-complete for BCCSP(A) modulo

It is not hard to see that IF1-2 are sound modulo $\precsim$ IF. For both axioms, the crucial observation for their soundness is that the impossible futures $(a, B)$ induced by $a(x+y)$ are included in those of $a x$. To give some intuition for the ground-completeness proof, we first present an example.

Example 5.2. Let $p=a\left(a \mathbf{0}+a^{2} \mathbf{0}\right)+a^{4} \mathbf{0}$ and $q=a\left(a \mathbf{0}+a^{3} \mathbf{0}\right)+a^{3} \mathbf{0}$. It is not hard to see that $p \precsim_{\text {IF }} q$. However, neither $a\left(a \mathbf{0}+a^{2} \mathbf{0}\right) \precsim_{\text {IF }} a\left(a \mathbf{0}+a^{3} \mathbf{0}\right)$ nor $a\left(a \mathbf{0}+a^{2} \mathbf{0}\right) \precsim_{\text {IF }} a^{3} \mathbf{0}$ holds. In order to derive $p \preccurlyeq q$, we therefore first derive $q \approx p+q$, and next $p \preccurlyeq p+q$.

In general, to derive a sound closed inequation $p \preccurlyeq q$, first we derive $q \approx \mathbb{S}(q)$ (see Lem. 5.5), where $\mathbb{S}(q)$ contains for every $a \in \mathcal{I}(q)$ a "saturated" $a$-summand (see Def. 5.3). (In Ex. 5.2 , this saturated summand would have the form $a\left(a \mathbf{0}+a^{2} \mathbf{0}+a^{3} \mathbf{0}+a\left(a \mathbf{0}+a^{2} \mathbf{0}\right)\right)$.) Then, in the proof of Thm. 5.1, we derive $\Psi+\mathbb{S}(q) \approx \mathbb{S}(q)$ (equation (5.1)), $p \preccurlyeq \Psi$ (equation (5.2) ) and $p \preccurlyeq p+q$ (equation (5.3) ), where the closed term $\Psi$ is built from many "semisaturated" summands (like, in Ex. $5.2, p$ ). These results together provide the desired proof (see the last line of the proof of Thm. 5.1).

In the remainder of this section, $a p^{\prime} \Subset p$ denotes that $a p^{\prime}$ is a summand of $p$.

Definition 5.3. For each closed term $q$, the closed term $\mathbb{S}(q)$ is defined recursively on the depth of $q$ as follows:

$$
\mathbb{S}(q)=q+\sum_{a \in \mathcal{I}(q)} a\left(\mathbb{S}\left(\sum_{a q^{\prime} \Subset q} q^{\prime}\right)\right)
$$

Example 5.4. If $q=a(b(c \mathbf{0}+d \mathbf{0})+b e \mathbf{0})+a f \mathbf{0}$, then $\mathbb{S}(q)=a(b(c \mathbf{0}+d \mathbf{0})+b e \mathbf{0})+a f \mathbf{0}+$ $a(b(c \mathbf{0}+d \mathbf{0})+b e \mathbf{0}+f \mathbf{0}+b(c \mathbf{0}+d \mathbf{0}+e \mathbf{0}))$.

Lemma 5.5. For each closed term $q, \mathrm{~A} 1-4+\mathrm{IF} 1-2 \vdash q \approx \mathbb{S}(q)$. 
Proof. By induction on the depth of $q$. For any $a \in \mathcal{I}(q)$,

$$
q \approx q+a\left(\sum_{a q^{\prime} \Subset q} q^{\prime}\right) \approx q+a\left(\mathbb{S}\left(\sum_{a q^{\prime} \Subset q} q^{\prime}\right)\right)
$$

The first derivation step uses IF2, and the second induction. Hence, summing up over all $a \in \mathcal{I}(q)$,

$$
q \approx q+\sum_{a \in \mathcal{I}(q)} a\left(\mathbb{S}\left(\sum_{a q^{\prime} \Subset q} q^{\prime}\right)\right)=\mathbb{S}(q)
$$

For any closed term $q$ and $a \in \mathcal{I}(q)$, the closed term $q_{a}$ is obtained by summing over all closed terms $q^{\prime}$ such that $q \stackrel{a}{\rightarrow} q^{\prime}$, and then applying the saturation from Def. 5.3 . The definition and lemma below generalize this idea to terms $q_{a_{1} \cdots a_{\ell}}$ with $a_{1} \cdots a_{\ell} \in \mathcal{T}(q)$. The auxiliary terms $q_{a_{1} \cdots a_{\ell}}$ will only be used in the derivation of equation (5.1) within the proof of Thm. 5.1.

Definition 5.6. Given a closed term $q$ and a trace $a_{1} \cdots a_{\ell}$ of $q$.

$$
Q_{a_{1} \cdots a_{\ell}}=\left\{q_{\ell} \mid \text { there exists a sequence of transitions } q \stackrel{a_{7}}{\rightarrow} q_{1} \cdots \stackrel{a_{\ell}}{\rightarrow} q_{\ell}\right\}
$$

and

$$
q_{a_{1} \cdots a_{\ell}}=\mathbb{S}\left(\sum_{q_{\ell} \in Q_{a_{1} \cdots a_{\ell}}} q_{\ell}\right)
$$

Note that $q_{\varepsilon}=\mathbb{S}(q)$. We prove some basic properties for the terms $q_{a_{1} \cdots a_{\ell}}$.

Lemma 5.7. Given a closed term $q$, and a completed trace $a_{1} \cdots a_{d}$ of $q$. Then, for each $0 \leq \ell<d$,

- $q_{a_{1} \cdots a_{\ell}} \stackrel{a_{\ell+1}}{\rightarrow} q_{a_{1} \cdots a_{\ell+1}} ;$ and

- $q_{a_{1} \cdots a_{\ell}} \stackrel{a_{\ell+1}}{\rightarrow} q_{\ell+1}$ for all $q_{\ell+1} \in Q_{a_{1} \cdots a_{\ell+1}}$.

Proof. Clearly, $q_{\ell+1} \in Q_{a_{1} \cdots a_{\ell+1}}$ iff there exists some $q_{\ell} \in Q_{a_{1} \cdots a_{\ell}}$ such that $q_{\ell} \stackrel{a_{\ell+1}}{\rightarrow} q_{\ell+1}$. And since $a_{1} \cdots a_{\ell+1}$ is a trace of $q, a_{\ell+1} \in \mathcal{I}\left(q_{\ell}\right)$ for some $q_{\ell} \in Q_{a_{1} \cdots a_{\ell}}$. So by Def. 5.3.

$$
q_{a_{1} \cdots a_{\ell}}=\mathbb{S}\left(\sum_{q_{\ell} \in Q_{a_{1} \cdots a_{\ell}}} q_{\ell}\right) \stackrel{a_{\ell+1}}{\rightarrow} \mathbb{S}\left(\sum_{q_{\ell+1} \in Q_{a_{1} \cdots a_{\ell+1}}} q_{\ell+1}\right)=q_{a_{1} \cdots a_{\ell+1}}
$$

Moreover, for all $q_{\ell+1} \in Q_{a_{1} \cdots a_{\ell+1}}$ we have $\sum_{q_{\ell} \in Q_{a_{1} \cdots a_{\ell}}} q_{\ell} \stackrel{a_{\ell+1}}{\rightarrow} q_{\ell+1}$. Hence, by Def. 5.3 .

$$
\left[q_{a_{1} \cdots a_{\ell}}=\mathbb{S}\left(\sum_{q_{\ell} \in Q_{a_{1} \cdots a_{\ell}}} q_{\ell}\right) \stackrel{a_{\ell+1}}{\rightarrow} q_{\ell+1}\right.
$$

We now embark on proving the promised ground-completeness result in Thm. 5.1.

Proof. Suppose $p \precsim$ IF $q$. We derive $p \preccurlyeq q$ using induction on the depth of $p$. If $p=\mathbf{0}$, then clearly $q=\mathbf{0}$, and we are done. So assume $p \neq \mathbf{0}$.

We call a sequence $a_{1} p_{1} \cdots a_{k} p_{k}$ a completed path of a closed term $p_{0}$ if $p_{0} \stackrel{a_{1}}{\rightarrow} p_{1} \cdots \stackrel{a_{k}}{\rightarrow} p_{k}$ with $\mathcal{I}\left(p_{k}\right)=\emptyset$. Let $\mathcal{C P}(p)$ denote the set of completed paths of $p$, ranged over by $\pi$. Consider any $\pi=a_{1} p_{1} \cdots a_{d} p_{d}$ in $\mathcal{C P}(p)$. Since $p \neq \mathbf{0}$, we have $d \geq 1$. We recursively construct closed terms $\psi_{\ell}^{\pi}$, where $\ell$ counts down from $d$ to 1 . For the base case we define $\psi_{d}^{\pi}=\mathbf{0}$. Now let 
$1 \leq \ell<d$. Since $p \stackrel{a_{1} \cdots a_{\ell}}{\rightarrow} p_{\ell}$ and $p \precsim$ IF $q$, there exists a sequence of transitions $q \stackrel{a_{1} \cdots a_{\ell}}{\rightarrow} q_{\ell}$ such that $\mathcal{T}\left(q_{\ell}\right) \subseteq \mathcal{T}\left(p_{\ell}\right)$. Given the choice of $\pi$ and $\ell$, we pick such a $q_{\ell}$ and call it $q_{\ell}^{\pi}$. Now define

$$
\psi_{\ell}^{\pi}=q_{\ell}^{\pi}+a_{\ell+1} \psi_{\ell+1}^{\pi}
$$

We prove, by induction on $d-\ell$, that for all $1 \leq \ell \leq d$,

$$
\mathcal{T}\left(\psi_{\ell}^{\pi}\right) \subseteq \mathcal{T}\left(p_{\ell}\right)
$$

The base case is trivial, since $\mathcal{T}\left(\psi_{d}^{\pi}\right)=\emptyset$. Now let $1 \leq \ell<d$. By induction, $\mathcal{T}\left(\psi_{\ell+1}^{\pi}\right) \subseteq$ $\mathcal{T}\left(p_{\ell+1}\right)$. Moreover, $p_{\ell} \stackrel{a_{\ell+1}}{\rightarrow} p_{\ell+1}$, so $\mathcal{T}\left(a_{\ell+1} \psi_{\ell+1}^{\pi}\right) \subseteq \mathcal{T}\left(p_{\ell}\right)$. Hence, $\mathcal{T}\left(\psi_{\ell}^{\pi}\right)=\mathcal{T}\left(q_{\ell}^{\pi}\right) \cup$ $\mathcal{T}\left(a_{\ell+1} \psi_{\ell+1}^{\pi}\right) \subseteq \mathcal{T}\left(p_{\ell}\right)$.

We now derive three (in)equations that together yield the desired result. First, we derive by induction on $d-\ell$, for all $1 \leq \ell \leq d$,

$$
a_{\ell} \psi_{\ell}^{\pi}+q_{a_{1} \cdots a_{\ell-1}} \approx q_{a_{1} \cdots a_{\ell-1}}
$$

In the base case, since $\psi_{d}^{\pi}=\mathbf{0} \in Q_{a_{1} \cdots a_{d}}$ (see Def. 5.6), this is a direct consequence of the second item in Lem. 5.7. Now let $1 \leq \ell<d$.

$$
\begin{array}{rlr} 
& a_{\ell} \psi_{\ell}^{\pi}+q_{a_{1} \cdots a_{\ell-1}} \\
= & a_{\ell}\left(q_{\ell}^{\pi}+a_{\ell+1} \psi_{\ell+1}^{\pi}\right)+q_{a_{1} \cdots a_{\ell-1}}+a_{\ell} q_{\ell}^{\pi}+a_{\ell} q_{a_{1} \cdots a_{\ell}} & \text { (Lem. 5.7) } \\
\approx & a_{\ell}\left(q_{\ell}^{\pi}+a_{\ell+1} \psi_{\ell+1}^{\pi}\right)+q_{a_{1} \cdots a_{\ell-1}}+a_{\ell} q_{\ell}^{\pi}+a_{\ell}\left(a_{\ell+1} \psi_{\ell+1}^{\pi}+q_{a_{1} \cdots a_{\ell}}\right) & \text { (induction) } \\
\approx & q_{a_{1} \cdots a_{\ell-1}}+a_{\ell} q_{\ell}^{\pi}+a_{\ell}\left(a_{\ell+1} \psi_{\ell+1}^{\pi}+q_{a_{1} \cdots a_{\ell}}\right) & \text { (IF2) } \\
\approx & q_{a_{1} \cdots a_{\ell-1}}+a_{\ell} q_{\ell}^{\pi}+a_{\ell} q_{a_{1} \cdots a_{\ell}} & \text { (induction) } \\
= & q_{a_{1} \cdots a_{\ell-1}} & \text { (Lem. 5.7) }
\end{array}
$$

In the end, for $\ell=1$, we have derived $a_{1} \psi_{1}^{\pi}+q_{\varepsilon} \approx q_{\varepsilon}$. In other words,

$$
a_{1} \psi_{1}^{\pi}+\mathbb{S}(q) \approx \mathbb{S}(q)
$$

Second, for every $a p^{\prime} \Subset p$,

$$
p^{\prime} \precsim \text { IF } \sum_{\pi \in \mathcal{C} \mathcal{P}\left(a p^{\prime}\right)} \psi_{1}^{\pi}
$$

Namely, consider any (possibly incomplete) path $\pi_{0}=a_{1} p_{1} \cdots a_{h} p_{h}$ of $a p^{\prime}$. Extend $\pi_{0}$ to some $\pi \in \mathcal{C P}\left(a p^{\prime}\right)$. Clearly, $\psi_{\ell}^{\pi} \stackrel{a_{\ell+1}}{\rightarrow} \psi_{\ell+1}^{\pi}$ for all $1 \leq \ell<h$, so $\psi_{1}^{\pi} \stackrel{a_{2} \cdots a_{h}}{\rightarrow} \psi_{h}^{\pi}$. Moreover, we proved that $\mathcal{T}\left(\psi_{h}^{\pi}\right) \subseteq \mathcal{T}\left(p_{h}\right)$.

So by induction on depth, for every $a p^{\prime} \Subset p$ we can derive

$$
p^{\prime} \preccurlyeq \sum_{\pi \in \mathcal{C} \mathcal{P}\left(a p^{\prime}\right)} \psi_{1}^{\pi}
$$

And thus, by IF1, we derive

$$
a p^{\prime} \preccurlyeq \sum_{\pi \in \mathcal{C} \mathcal{P}\left(a p^{\prime}\right)} a \psi_{1}^{\pi}
$$

Hence, summing over all summands $a p^{\prime}$ of $p$, we derive

$$
p \preccurlyeq \sum_{a \in \mathcal{I}(p)} \sum_{a p^{\prime} \Subset p} \sum_{\pi \in \mathcal{C} \mathcal{P}\left(a p^{\prime}\right)} a \psi_{1}^{\pi}
$$


Third, since $p \precsim$ IF $q$, clearly, for each $a \in \mathcal{I}(p)$,

$$
\sum_{a p^{\prime} \Subset p} p^{\prime} \precsim \mathrm{IF} \sum_{a q^{\prime} \Subset q} q^{\prime}
$$

So by induction on depth, for each $a \in \mathcal{I}(p)$ we can derive

$$
\sum_{a p^{\prime} \Subset p} p^{\prime} \preccurlyeq \sum_{a q^{\prime} \Subset q} q^{\prime}
$$

So by IF2 and IF1, and since $\mathcal{I}(p)=\mathcal{I}(q)$, we derive

$$
p \approx p+\sum_{a \in \mathcal{I}(p)} a\left(\sum_{a p^{\prime} \Subset p} p^{\prime}\right) \preccurlyeq p+\sum_{a \in \mathcal{I}(q)} a\left(\sum_{a q^{\prime} \Subset q} q^{\prime}\right) \preccurlyeq p+\sum_{a \in \mathcal{I}(q)} \sum_{a q^{\prime} \Subset q} a q^{\prime}
$$

That is, we have derived

$$
p \preccurlyeq p+q
$$

Finally, (5.3), Lem.5.5, (5.2) and (5.1) yield the derivation

$$
p \preccurlyeq p+q \approx p+\mathbb{S}(q) \preccurlyeq \sum_{a \in \mathcal{I}(p)} \sum_{a p^{\prime} \Subset p} \sum_{\pi \in \mathcal{C} \mathcal{P}\left(a p^{\prime}\right)} a \psi_{1}^{\pi}+\mathbb{S}(q) \approx \mathbb{S}(q) \approx q
$$

5.2. Weak impossible futures preorder. We now apply the link established in Sect. 3 to obtain a ground-complete axiomatization for $\operatorname{BCCS}(A)$ modulo $\precsim$ WIF. It consists of A1-4, WIF1-2 and W1 together with

$$
\begin{aligned}
& \text { IF1' } \quad \alpha(x+y) \preccurlyeq \alpha x+\alpha y \\
& \text { IF2 }^{\prime} \quad \alpha x+\alpha(y+z) \approx \alpha(x+y)+\alpha x+\alpha(y+z)
\end{aligned}
$$

It is not hard to see that this axiomatization is sound modulo $\precsim$ WIF.

Again, this axiomatization can be simplified. It turns out that IF1' and IF2' are redundant. Namely, by W1 and WIF1, $\alpha(x+y) \preccurlyeq \alpha(\tau x+\tau y) \approx \alpha x+\alpha y$. And by WIF1 and WIF2, $\alpha x+\alpha(y+z) \approx \alpha(\tau x+\tau(y+z))=\alpha(\tau x+\tau(y+z+y)+\tau(y+z)) \approx$ $\alpha(\tau x+y+\tau(y+z)) \approx \alpha(\tau(x+y)+\tau x+\tau(y+z)) \approx \alpha(\tau(x+y)+\tau(\tau x+\tau(y+z))) \approx$ $\alpha(x+y)+\alpha(\tau x+\tau(y+z)) \approx \alpha(x+y)+\alpha x+\alpha(y+z)$. Furthermore, WIF2 can be replaced by WIF2'. Namely, by WIF2', $\tau(x+y)+\tau x \preccurlyeq \tau x+y$; and by WIF2' and W1, $\tau x+y=\tau x+\tau(x+x)+y \preccurlyeq \tau x+x+y \preccurlyeq \tau x+\tau(x+y)$.

The ground-completeness claim in the following corollary is an immediate consequence of Thm. 5.1 together with Thm. 3.4.

Corollary 5.8. A1-4+WIF1+WIF2'+W1 is sound and ground-complete for $B C C S(A)$ modulo $\precsim$ WIF .

This result was already proved in [VM01] (for a slightly more complicated axiomatization). There an intricate ground-completeness proof was given, which relies heavily on the presence of $\tau$. 
5.3. Weak impossible futures equivalence. We now prove that there does not exist a finite, sound, ground-complete axiomatization for $\operatorname{BCCS}(A)$ modulo $\simeq_{\mathrm{WIF}}$. The cornerstone for this negative result is the following infinite family of closed equations. Pick an $a \in A$. For each $m \geq 0$,

$$
\tau a^{2 m} \mathbf{0}+\tau\left(a^{m} \mathbf{0}+a^{2 m} \mathbf{0}\right) \approx \tau\left(a^{m} \mathbf{0}+a^{2 m} \mathbf{0}\right)
$$

is sound modulo $\simeq_{\mathrm{WIF}}$. We start with a few lemmas.

Lemma 5.9. If $t \precsim$ WIF $u$ and $t \Rightarrow \stackrel{\tau}{\rightarrow} t^{\prime}$, then there is a term $u^{\prime}$ with $u \Rightarrow \stackrel{\tau}{\rightarrow} u^{\prime}$ and $\operatorname{var}\left(u^{\prime}\right) \subseteq \operatorname{var}\left(t^{\prime}\right)$.

Proof. Let $t \Rightarrow \stackrel{\tau}{\rightarrow} t^{\prime}$. Pick an $a \in A$ and $m>d e p t h_{w}(t)$, and consider the closed substitution $\rho$ defined by $\rho(x)=\mathbf{0}$ if $x \in \operatorname{var}\left(t^{\prime}\right)$ and $\rho(x)=a^{m} \mathbf{0}$ if $x \notin \operatorname{var}\left(t^{\prime}\right)$. Since $\rho(t) \Rightarrow \rho\left(t^{\prime}\right)$ with $\operatorname{depth}_{w}\left(\rho\left(t^{\prime}\right)\right)=$ depth $_{w}\left(t^{\prime}\right)<m$, and $\rho(t) \precsim$ WIF $\rho(u)$, clearly $\rho(u) \Rightarrow q$ for some $q$ with $\operatorname{depth}_{w}(q)<m$. From the definition of $\rho$ it then follows that $u \Rightarrow u^{\prime}$ for some $u^{\prime}$ with $\operatorname{var}\left(u^{\prime}\right) \subseteq \operatorname{var}\left(t^{\prime}\right)$. In case $u \Rightarrow \stackrel{\tau}{\rightarrow} u^{\prime}$ we are done, so assume $u^{\prime}=u$. Let $\sigma$ be the closed substitution with $\sigma(x)=\mathbf{0}$ for all $x \in V$. Since $\sigma(t) \stackrel{\tau}{\rightarrow}$ and $t \precsim$ WIF $u$, we have $\sigma(u) \stackrel{\tau}{\rightarrow}$, so $u \stackrel{\tau}{\rightarrow} u^{\prime \prime}$ for some $u^{\prime \prime}$. And $\operatorname{var}\left(u^{\prime \prime}\right) \subseteq \operatorname{var}(u)=\operatorname{var}\left(u^{\prime}\right) \subseteq \operatorname{var}\left(t^{\prime}\right)$.

Lemma 5.10. Suppose that for some terms $t, u$, closed substitution $\sigma$, action $a$ and $m>0$ :

(1) $t \simeq_{\mathrm{WIF}} u$;

(2) $m>\operatorname{depth}_{w}(u)$

(3) $\mathcal{W C T}(\sigma(u)) \subseteq\left\{a^{m}, a^{2 m}\right\}$; and

(4) there is a closed term $p^{\prime}$ such that $\sigma(t) \Rightarrow \stackrel{\tau}{\rightarrow} p^{\prime}$ and $\mathcal{W C T}\left(p^{\prime}\right)=\left\{a^{2 m}\right\}$.

Then there is a closed term $q^{\prime}$ such that $\sigma(u) \Rightarrow \stackrel{\tau}{\rightarrow} q^{\prime}$ and $\mathcal{W C T}\left(q^{\prime}\right)=\left\{a^{2 m}\right\}$.

Proof. We note that if $p \precsim$ WIF $q$, then $\mathcal{W C T}(p) \subseteq \mathcal{W C T}(q)$. Namely, a process has a weak completed trace $a_{1} \cdots a_{k}$ iff it has a weak impossible future $\left(a_{1} \cdots a_{k}, A\right)$.

According to proviso (4) of the lemma, two cases can be distinguished: the trace $\sigma(t) \Rightarrow \stackrel{\tau}{\rightarrow} p^{\prime}$ visits a variable in $t$ or not.

- $t \Rightarrow \stackrel{\tau}{\rightarrow} t^{\prime}$ for some $t^{\prime}$ with $\sigma\left(t^{\prime}\right)=p^{\prime}$. Since $\operatorname{depth}_{w}\left(t^{\prime}\right) \leq \operatorname{depth}_{w}(t)=\operatorname{depth}_{w}(u)<m$ and $\mathcal{W C} \mathcal{T}\left(p^{\prime}\right)=\left\{a^{2 m}\right\}$, for any $y \in \operatorname{var}\left(t^{\prime}\right)$ either $\sigma(y)=\mathbf{0}$ or $\mathcal{W C} \mathcal{T}(\sigma(y))=\left\{a^{2 m}\right\}$. Since $t \simeq$ WIF $u$, by Lem. 5.9, $u \Rightarrow \stackrel{\tau}{\rightarrow} u^{\prime}$ for some $u^{\prime}$ with $\operatorname{var}\left(u^{\prime}\right) \subseteq \operatorname{var}\left(t^{\prime}\right)$. Hence, for any $y \in \operatorname{var}\left(u^{\prime}\right)$ either $\sigma(y)=\mathbf{0}$ or $\mathcal{W C T}(\sigma(y))=\left\{a^{2 m}\right\}$. Since depth ${ }_{w}\left(u^{\prime}\right) \leq \operatorname{depth}_{w}(u)<m$, it follows that $a^{m} \notin \mathcal{W C T}\left(\sigma\left(u^{\prime}\right)\right)$. Since $\mathcal{W C T}(\sigma(u)) \subseteq\left\{a^{m}, a^{2 m}\right\}$, we conclude that $\mathcal{W C T}\left(\sigma\left(u^{\prime}\right)\right)=\left\{a^{2 m}\right\}$. And $u \Rightarrow \stackrel{\tau}{\rightarrow} u^{\prime}$ implies $\sigma(u) \Rightarrow \stackrel{\tau}{\rightarrow} \sigma\left(u^{\prime}\right)$.

- $t \Rightarrow t^{\prime}+x$ for some $t^{\prime}$ and $x$ with $\sigma(x) \Rightarrow \stackrel{\tau}{\rightarrow} p^{\prime}$. Consider the closed substitution $\rho$ defined by $\rho(x)=a^{m} \mathbf{0}$ and $\rho(y)=\mathbf{0}$ for any $y \neq x$. Then $a^{m} \in \mathcal{W C T}(\rho(t))=\mathcal{W C} \mathcal{T}(\rho(u))$. Since $\operatorname{depth}_{w}(u)<m$, in view of the definition of $\rho$ this implies that $u \Rightarrow u^{\prime}+x$ for some $u^{\prime}$. Hence $\sigma(u) \Rightarrow \stackrel{\tau}{\rightarrow} p^{\prime}$.

Proposition 5.11. Suppose that for a sound axiomatization $E$ for $\operatorname{BCCS}(A)$ modulo $\simeq_{\mathrm{WIF}}$, closed terms $p, q$, closed substitution $\sigma$, action $a$ and $m>0$ :

(1) $E \vdash p \approx q$ or $E \vdash q \approx p$;

(2) $m>\max \left\{\operatorname{depth}_{w}(u) \mid t \approx u \in E\right\}$;

(3) $\mathcal{W C T}(q) \subseteq\left\{a^{m}, a^{2 m}\right\}$; and

(4) there is a closed term $p^{\prime}$ such that $p \Rightarrow \stackrel{\tau}{\rightarrow} p^{\prime}$ and $\mathcal{W C T}\left(p^{\prime}\right)=\left\{a^{2 m}\right\}$.

Then there is a closed term $q^{\prime}$ such that $q \Rightarrow \stackrel{\tau}{\rightarrow} q^{\prime}$ and $\mathcal{W C T}\left(q^{\prime}\right)=\left\{a^{2 m}\right\}$. 
Proof. By induction on a derivation of $E \vdash p \approx q$ or $E \vdash q \approx p$. We only consider $E \vdash p \approx q$; the proof for $E \vdash q \approx p$ is symmetrical.

The cases where $E \vdash p \approx q$ is derived by reflexivity (i.e., $q=p$ ) or symmetry (i.e., $E \vdash p \approx q$ because $E \vdash q \approx p)$ are trivial. We focus on the other cases.

- Suppose $E \vdash p \approx q$ because $\sigma(t)=p$ and $\sigma(u)=q$ for some $t \approx u \in E$ or $u \approx t \in E$ and closed substitution $\sigma$. The claim then follows by Lem. 5.10 .

- Suppose $E \vdash p \approx q$ because $E \vdash p \approx r$ and $E \vdash r \approx q$ for some $r$. Since $r \simeq_{\text {WIF }} q$, by proviso $(3), \mathcal{W C T}(r)=\mathcal{W C T}(q) \subseteq\left\{a^{m}, a^{2 m}\right\}$. Since $p \Rightarrow \stackrel{\tau}{\rightarrow} p^{\prime}$ and $\mathcal{W C T}\left(p^{\prime}\right)=\left\{a^{2 m}\right\}$, by induction there is an $r^{\prime}$ such that $r \Rightarrow \stackrel{\tau}{\rightarrow} r^{\prime}$ and $\mathcal{W C T}\left(r^{\prime}\right)=\left\{a^{2 m}\right\}$. Hence, again by induction there is a $q^{\prime}$ such that $q \Rightarrow \stackrel{\tau}{\rightarrow} q^{\prime}$ and $\mathcal{W C T}\left(q^{\prime}\right)=\left\{a^{2 m}\right\}$.

- Suppose $E \vdash p \approx q$ because $p=p_{1}+p_{2}$ and $q=q_{1}+q_{2}$ with $E \vdash p_{1} \approx q_{1}$ and $E \vdash p_{2} \approx q_{2}$. Since $p \Rightarrow \stackrel{\tau}{\rightarrow} p^{\prime}$, we have $p_{i} \Rightarrow \stackrel{\tau}{\rightarrow} p^{\prime}$ for an $i \in\{1,2\}$. And $\mathcal{W C T}\left(q_{i}\right) \subseteq \mathcal{W C T}(q) \subseteq$ $\left\{a^{m}, a^{2 m}\right\}$. Hence, by induction there is a $q^{\prime}$ such that $q_{i} \Rightarrow \stackrel{\tau}{\rightarrow} q^{\prime}$ and $\mathcal{W C T}\left(q^{\prime}\right)=\left\{a^{2 m}\right\}$. And $q_{i} \Rightarrow \stackrel{\tau}{\rightarrow} q^{\prime}$ implies $q \Rightarrow \stackrel{\tau}{\rightarrow} q^{\prime}$.

- Suppose $E \vdash p \approx q$ because $p=\alpha p_{1}$ and $q=\alpha q_{1}$ with $E \vdash p_{1} \approx q_{1}$. By proviso (4), $\alpha=\tau$, and either $\mathcal{W C} \mathcal{T}\left(p_{1}\right)=\left\{a^{2 m}\right\}$ or there is a $p^{\prime}$ such that $p_{1} \Rightarrow \stackrel{\tau}{\rightarrow} p^{\prime}$ and $\mathcal{W C T}\left(p^{\prime}\right)=\left\{a^{2 m}\right\}$. In the first case, $\mathcal{W C T}\left(q_{1}\right)=\mathcal{W C T}\left(p_{1}\right)=\left\{a^{2 m}\right\}$; and $q \Rightarrow \stackrel{\tau}{\rightarrow} q_{1}$. In the second case, $p \Rightarrow \stackrel{\tau}{\rightarrow} p^{\prime}$ and $\mathcal{W C T}\left(q_{1}\right)=\mathcal{W C T}(q) \subseteq\left\{a^{m}, a^{2 m}\right\}$. So by induction there is a $q^{\prime}$ such that $q_{1} \Rightarrow \stackrel{\tau}{\rightarrow} q^{\prime}$ and $\mathcal{W C T}\left(q^{\prime}\right)=\left\{a^{2 m}\right\}$. And $q_{1} \Rightarrow \stackrel{\tau}{\rightarrow} q^{\prime}$ implies $q \Rightarrow \stackrel{\tau}{\rightarrow} q^{\prime}$.

Theorem 5.12. There is no finite, sound, ground-complete axiomatization for BCCS(A) modulo $\simeq$ WIF

Proof. Let $E$ be a finite axiomatization over $\operatorname{BCCS}(A)$ that is sound modulo $\simeq_{\text {WIF }}$. Pick an $m$ greater than the weak depth of any term in $E$. Consider the closed equation $\tau a^{2 m} \mathbf{0}+\tau\left(a^{m} \mathbf{0}+\right.$ $\left.a^{2 m} \mathbf{0}\right) \approx \tau\left(a^{m} \mathbf{0}+a^{2 m} \mathbf{0}\right)$, which is sound modulo $\simeq_{\mathrm{WIF}}$. We have $\mathcal{W C T}\left(\tau\left(a^{m} \mathbf{0}+a^{2 m} \mathbf{0}\right)\right)=$ $\left\{a^{m}, a^{2 m}\right\}$ and $\tau a^{2 m} \mathbf{0}+\tau\left(a^{m} \mathbf{0}+a^{2 m} \mathbf{0}\right) \stackrel{\tau}{\rightarrow} a^{2 m} \mathbf{0}$. However, clearly there is no closed term $r$ such that $\tau\left(a^{m} \mathbf{0}+a^{2 m} \mathbf{0}\right) \Rightarrow \stackrel{\tau}{\rightarrow} r$ and $\mathcal{W C T}(r)=\left\{a^{2 m}\right\}$. So according to Prop. 5.11, this closed equation cannot be derived from $E$.

Remark 5.13. To explain why there is no counterpart of Thm. 5.12 for $\precsim$ WIF, we note that Prop. 5.11 does not hold if its first requirement is changed into $E \vdash p \preccurlyeq q$. Namely, the proof regarding the congruence rule for $\tau$.- in Prop. 5.11 fails for $\precsim$ WIF.

For example, for each $m \geq 0$,

$$
\tau a^{2 m} \mathbf{0} \precsim \mathrm{WIF} \quad \tau\left(a^{2 m} \mathbf{0}+a^{m} \mathbf{0}\right)
$$

These relations satisfy the third and fourth requirement of Prop. 5.11. However, it is not the case that $\tau\left(a^{2 m} \mathbf{0}+a^{m} \mathbf{0}\right) \Rightarrow \stackrel{\tau}{\rightarrow} q^{\prime}$ with $\mathcal{W C T}\left(q^{\prime}\right)=\left\{a^{2 m}\right\}$.

We note that these relations can all be derived by means of IF1':

$$
\begin{aligned}
& \tau a^{2 m} \mathbf{0}=\tau\left(a^{m}\left(a^{m}+\mathbf{0}\right)\right) \preccurlyeq \tau\left(a^{m-1}\left(a^{m+1} \mathbf{0}+a \mathbf{0}\right)\right) \\
& \preccurlyeq \tau\left(a^{m-2}\left(a^{m+2} \mathbf{0}+a^{2} \mathbf{0}\right)\right) \preccurlyeq \cdots \preccurlyeq \tau\left(a^{2 m} \mathbf{0}+a^{m} \mathbf{0}\right)
\end{aligned}
$$


5.4. Concrete impossible futures equivalence. There also does not exist a finite, sound, ground-complete axiomatization for $\operatorname{BCCSP}(A)$ modulo $\simeq_{\mathrm{IF}}$. The cornerstone for this negative result is the following infinite family of closed equations. For each $m \geq 0$,

$$
a^{2 m+1} \mathbf{0}+a\left(a^{m} \mathbf{0}+a^{2 m} \mathbf{0}\right) \approx a\left(a^{m} \mathbf{0}+a^{2 m} \mathbf{0}\right)
$$

is sound modulo $\simeq_{\mathrm{IF}}$. The proof of the following theorem, which can be found in [CF08, is very similar to the proof of Thm. 5.12, and is therefore omitted here.

Theorem 5.14. There is no finite, sound, ground-complete axiomatization for $\operatorname{BCCSP}(A)$ modulo $\simeq_{\mathrm{IF}}$.

We note that this negative result for $\simeq_{\mathrm{IF}}$ is not a direct consequence of the corresponding negative result for $\simeq_{\text {WIF }}$ (Thm. 5.12) together with Thm. 3.6. Firstly, Thm. 3.6 disregards soundness, meaning that a finite, sound, ground-complete axiomatization $E$ for $\operatorname{BCCSP}(A)$ modulo $\simeq_{\mathrm{IF}}$ could in theory generate an unsound axiomatization $\mathcal{A}(E)$ for $\operatorname{BCCS}(A)$ modulo $\simeq_{\text {WIF }}$. Secondly, Thm. 3.6 only applies to safe axiomatizations $E$.

Interestingly, Thm. 5.14 would be a direct consequence of Thm. 5.12 together with the alternative method described in Remark 3.11. Namely, that method does imply soundness of the generated axiomatization, and is not restricted to safe axiomatizations.

The infinite families of equations that are used to prove Thm. 5.12 and 5.14 are also sound modulo weak and concrete 2-nested simulation equivalence, respectively. Therefore these negative results apply to all BCCS- and BCCSP-congruences that are at least as fine as impossible futures equivalence and at least as coarse as 2-nested simulation equivalence. This infers some results from [AFGI04], where among others concrete 2-nested simulation and possible futures equivalence were considered.

\section{6. $\omega$-Completeness for the Impossible Futures Preorder}

This section deals with the existence of $\omega$-complete axiomatizations in the context of the impossible futures preorder.

6.1. Inverted substitutions. Groote [G90] introduced the technique of inverted substitutions to prove that an equational axiomatization is $\omega$-complete. Here we adapt his technique to make it suitable for inequational axiomatizations. Let $\mathrm{T}(\Sigma)$ and $\mathbb{T}(\Sigma)$ denote the sets of closed and open terms, respectively, over some signature $\Sigma$.

Theorem 6.1. Consider an inequational axiomatization $E$ over $\Sigma$. Suppose that for each inequation $t \preccurlyeq u$ of which all closed instances can be derived from $E$, there are a mapping $R: \mathrm{T}(\Sigma) \rightarrow \mathbb{T}(\Sigma)$ and a closed substitution $\rho$ such that:

(1) $E \vdash t \preccurlyeq R(\rho(t))$ and $E \vdash R(\rho(u)) \preccurlyeq u$;

(2) $E \vdash R(\sigma(v)) \preccurlyeq R(\sigma(w))$ for each $v \preccurlyeq w \in E$ and closed substitution $\sigma$; and

(3) for each function symbol $f$ (with arity $n$ ) in the signature, and all closed terms $p_{1}, \ldots, p_{n}, q_{1}, \ldots, q_{n}$ :

$$
\begin{aligned}
E \cup\left\{p_{i} \preccurlyeq q_{i}, R\left(p_{i}\right) \preccurlyeq R\left(q_{i}\right) \mid i=1, \ldots, n\right\} & \vdash \\
& R\left(f\left(p_{1}, \ldots, p_{n}\right)\right) \preccurlyeq R\left(f\left(q_{1}, \ldots, q_{n}\right)\right)
\end{aligned}
$$

Then $E$ is w-complete. 
The underlying idea of Thm. 6.1 is that each derivation of a closed inequation $p \preccurlyeq q$ from $E$ induces, by requirements (2) and (3), a derivation of the open inequation $R(p) \preccurlyeq R(q)$ from $E$. Since $E \vdash \rho(t) \preccurlyeq \rho(u)$, it follows using requirement (1) that $E \vdash t \preccurlyeq R(\rho(t)) \preccurlyeq$ $R(\rho(u)) \preccurlyeq u$. We now proceed to prove this theorem.

Proof. Let $t, u$ be terms such that for each closed substitution $\sigma$,

$$
\sigma(t) \preccurlyeq \sigma(u)
$$

By assumption, there are a mapping $R: \mathrm{T}(\Sigma) \rightarrow \mathbb{T}(\Sigma)$ and a closed substitution $\rho$ such that the three requirements of Thm. 6.1 are satisfied. We have to prove that $E \vdash t \preccurlyeq u$. This is an immediate corollary of the following claim, for all closed terms $p, q$ :

$$
E \vdash p \preccurlyeq q \Longrightarrow E \vdash R(p) \preccurlyeq R(q)
$$

Namely, by assumption, $E \vdash \rho(t) \preccurlyeq \rho(u)$, and then the claim above implies that $E \vdash$ $R(\rho(t)) \preccurlyeq R(\rho(u))$. So by requirement (1) of Thm. 6.1, $E \vdash t \preccurlyeq u$.

We prove the claim by induction on a derivation of $E \vdash p \preccurlyeq q$. We have to check the four kinds of inference rules.

- $p=q$. Then $R(p)=R(q)$.

- $p \preccurlyeq q$ is an instance of some $v \preccurlyeq w \in E$ and a closed substitution $\sigma$. By requirement (2) of Thm. 6.1. $E \vdash R(p) \preccurlyeq R(q)$.

- $E \vdash p \preccurlyeq q$ has been proved by $E \vdash p \preccurlyeq r$ and $E \vdash r \preccurlyeq q$, for some $r$. By induction, $E \vdash R(p) \preccurlyeq R(r)$ and $E \vdash R(r) \preccurlyeq R(q)$. So $E \vdash R(p) \preccurlyeq R(q)$.

- $p=f\left(p_{1}, \ldots, p_{n}\right)$ and $q=f\left(q_{1}, \ldots, q_{n}\right)$, and $E \vdash p \preccurlyeq q$ has been proved by $E \vdash p_{i} \preccurlyeq q_{i}$ for $i=1, \ldots, n$. By induction, $E \vdash R\left(p_{i}\right) \preccurlyeq R\left(q_{i}\right)$ for $i=1, \ldots, n$. So by requirement (3) of Thm.6.1, $E \vdash R\left(f\left(p_{1}, \ldots, p_{n}\right)\right) \preccurlyeq R\left(f\left(q_{1}, \ldots, q_{n}\right)\right)$.

6.2. Infinite alphabet. We show that the axiomatization consisting of A1-4+IF1-2 is $\omega$-complete, provided the alphabet is infinite. The proof is based on inverted substitutions.

Theorem 6.2. If $|A|=\infty$, then $\mathrm{A} 1-4+\mathrm{IF} 1-2$ is $\omega$-complete for $\operatorname{BCCSP}(A)$.

Proof. We define the closed substitution $\rho$ by $\rho(y)=a_{y} \mathbf{0}$, where $a_{y}$ is a distinct action in $A$ for each $y \in V$ that occurs in neither $t$ nor $u$. Such actions exist because $A$ is infinite. We define the mapping $R$ from closed to open $\operatorname{BCCSP}(A)$ terms as follows:

$$
\left\{\begin{array}{lll}
R(\mathbf{0}) & =\mathbf{0} & \text { if } a=a_{y} \text { for some } y \in V \\
R(a t) & =y & \text { if } a \neq a_{y} \text { for all } y \in V \\
R(a t) & =a R(t) & \\
R(t+u) & =R(t)+R(u) &
\end{array}\right.
$$

Consider a pair of $\operatorname{BCCSP}(A)$ terms $t, u$ such that all closed instances of $t \preccurlyeq u$ can be derived from A1-4+IF1-2. We check the three requirements of Thm.6.1.

(1) Since $t$ and $u$ do not contain actions of the form $a_{y}$, clearly $R(\rho(t))=t$ and $R(\rho(u))=u$.

(2) For A1-4, the proof is trivial, because for each of these four axioms $v \approx w, R(\sigma(v)) \approx$ $R(\sigma(w))$ is always a substitution instance of the axiom itself. We check the remaining cases IF1 and IF2. Let $\sigma$ be a closed substitution. With regard to IF1 we distinguish two cases. 
- $a=a_{y}$ for some $y \in V$. Then

$$
R\left(a_{y}\left(\sigma\left(x_{1}\right)+\sigma\left(x_{2}\right)\right)\right)=y=y+y=R\left(a_{y}\left(\sigma\left(x_{1}\right)\right)+a_{y}\left(\sigma\left(x_{2}\right)\right)\right) .
$$

$-a \neq a_{y}$ for all $y \in V$. Then using IF1 we derive

$$
\begin{aligned}
R\left(a\left(\sigma\left(x_{1}\right)+\sigma\left(x_{2}\right)\right)\right) & =a\left(R\left(\sigma\left(x_{1}\right)\right)+R\left(\sigma\left(x_{2}\right)\right)\right) \\
& \preccurlyeq a R\left(\sigma\left(x_{1}\right)\right)+a R\left(\sigma\left(x_{2}\right)\right) \\
& =R\left(a \sigma\left(x_{1}\right)+a \sigma\left(x_{2}\right)\right) .
\end{aligned}
$$

With regard to IF2 we distinguish two cases as well.

$-a=a_{y}$ for some $y \in V$. Then

$$
\begin{aligned}
R\left(a_{y} \sigma\left(x_{1}\right)+a_{y}\left(\sigma\left(x_{2}\right)+\sigma\left(x_{3}\right)\right)\right) & =y+y \\
& =y+y+y \\
& =R\left(a_{y}\left(\sigma\left(x_{1}\right)+\sigma\left(x_{2}\right)\right)+a_{y} \sigma\left(x_{1}\right)+a_{y}\left(\sigma\left(x_{2}\right)+\sigma\left(x_{3}\right)\right)\right) .
\end{aligned}
$$

$-a \neq a_{y}$ for all $y \in V$. Then using IF2 we derive

$$
\begin{aligned}
R\left(a \sigma\left(x_{1}\right)+a\left(\sigma\left(x_{2}\right)+\sigma\left(x_{3}\right)\right)\right)= & a R\left(\sigma\left(x_{1}\right)\right)+a\left(R\left(\sigma\left(x_{2}\right)\right)+R\left(\sigma\left(x_{3}\right)\right)\right) \\
\approx & a\left(R\left(\sigma\left(x_{1}\right)\right)+R\left(\sigma\left(x_{2}\right)\right)\right) \\
& +a R\left(\sigma\left(x_{1}\right)\right)+a\left(R\left(\sigma\left(x_{2}\right)\right)+R\left(\sigma\left(x_{3}\right)\right)\right) \\
= & R\left(a\left(\sigma\left(x_{1}\right)+\sigma\left(x_{2}\right)\right)+a \sigma\left(x_{1}\right)+a\left(\sigma\left(x_{2}\right)+\sigma\left(x_{3}\right)\right)\right) .
\end{aligned}
$$

(3) Consider the operator _ $+_{\text {_. From }} R\left(p_{1}\right) \preccurlyeq R\left(q_{1}\right)$ and $R\left(p_{2}\right) \preccurlyeq R\left(q_{2}\right)$ we derive

$$
R\left(p_{1}+p_{2}\right)=R\left(p_{1}\right)+R\left(p_{2}\right) \preccurlyeq R\left(q_{1}\right)+R\left(q_{2}\right)=R\left(q_{1}+q_{2}\right) .
$$

Consider the prefix operator $a_{-}$. We distinguish two cases.

- $a=a_{y}$ for some $y \in V$. Then $R\left(a_{y} p_{1}\right)=y=R\left(a_{y} q_{1}\right)$.

$-a \neq a_{y}$ for all $y \in V$. Then from $R\left(p_{1}\right) \preccurlyeq R\left(q_{1}\right)$ we derive

$$
R\left(a p_{1}\right)=a R\left(p_{1}\right) \preccurlyeq a R\left(q_{1}\right)=R\left(a q_{1}\right) .
$$

Corollary 6.3. If $|A|=\infty$, then $\mathrm{A} 1-4+\mathrm{WIF} 1+\mathrm{WIF} 2^{\prime}+\mathrm{W} 1$ is $\omega$-complete for $B C C S(A)$.

This result was already obtained in [VM01] (they do not refer to $\omega$-completeness explicitly, but their completeness proof works for open terms, see [VM01, Thm. 5]). Here it is a direct corollary of the link established in Sect. 3 , by the same reasoning as in Sect. 5.2 , 


\subsection{Finite alphabet.}

6.3.1. $1<|A|<\infty$. We prove that the inequational theory of $\operatorname{BCCS}(A)$ modulo $\precsim$ WIF does not have a finite basis in case of a finite alphabet $A$ with at least two elements. Pick an $a \in A$ and an $x \in V$. The cornerstone for this negative result is the following infinite family of inequations, for each $m \geq 0$ :

$$
\tau\left(a^{m} x\right)+\Phi_{m} \preccurlyeq \Phi_{m}
$$

with

$$
\Phi_{m}=\tau\left(a^{m} x+x\right)+\sum_{b \in A} \tau\left(a^{m} x+a^{m} b \mathbf{0}\right)
$$

We argue that these inequations are sound modulo $\precsim$ WIF. For any closed substitution $\rho$ we have $\mathcal{W} \mathcal{T}\left(\rho\left(\tau\left(a^{m} x\right)\right)\right) \subseteq \mathcal{W} \mathcal{T}\left(\rho\left(\Phi_{m}\right)\right)$ and $\rho\left(\Phi_{m}\right) \stackrel{\tau}{\rightarrow}$. To argue that $\rho\left(\tau\left(a^{m} x\right)+\Phi_{m}\right)$ and $\rho\left(\Phi_{m}\right)$ have the same weak impossible futures, it suffices to consider the transition $\rho\left(\tau\left(a^{m} x\right)+\Phi_{m}\right) \stackrel{\tau}{\rightarrow} a^{m} \rho(x)$. If $\rho(x)=\mathbf{0}$, then $\rho\left(\Phi_{m}\right) \stackrel{\tau}{\rightarrow} a^{m} \mathbf{0}+\mathbf{0}$ generates the same weak impossible futures $(\varepsilon, B)$. If, on the other hand, $b \in \mathcal{I}(\rho(x))$ for some $b \in A$, then $\mathcal{W} \mathcal{T}\left(a^{m} \rho(x)+a^{m} b \mathbf{0}\right)=\mathcal{W} \mathcal{T}\left(a^{m} \rho(x)\right)$, so $\rho\left(\Phi_{m}\right) \stackrel{\tau}{\rightarrow} a^{m} \rho(x)+a^{m} b \mathbf{0}$ generates the same weak impossible futures $(\varepsilon, B)$.

We extend the notions of weak traces from closed to open terms, allowing weak traces of the form $a_{1} \cdots a_{k} x \in A^{*} V$. This is done by treating each variable occurrence $x$ in a term as if it were a subterm $x \mathbf{0}$ with $x$ a concrete action. For example, under this convention the weak completed traces of $\Phi_{m}$ are $a^{m} x, x$ and $a^{m} b$ for all $b \in A$. We write $\mathcal{W} \mathcal{T}_{V}(t)$ for the set of weak traces of $t$ that end with a variable. So $\mathcal{W} \mathcal{T}_{V}\left(\Phi_{m}\right)=\left\{a^{m} x, x\right\}$. Note that $\mathcal{W} \mathcal{T}_{V}(t) \subseteq \mathcal{W} \mathcal{T}_{V}(u)$ implies $\mathcal{W} \mathcal{T}_{V}(\sigma(t)) \subseteq \mathcal{W} \mathcal{T}_{V}(\sigma(u))$ for any terms $u, v$ and substitution $\sigma$.

Remark 6.4. Let $m>\operatorname{depth}_{w}(t)$ and $\rho$ a closed substitution. Then $a_{1} \cdots a_{m} \in \mathcal{W T}(\rho(t))$ iff there are a $k<m$ and $y \in V$ such that $a_{1} \cdots a_{k} y \in \mathcal{W} \mathcal{T}_{V}(t)$ and $a_{k+1} \cdots a_{m} \in \mathcal{W} \mathcal{T}(\rho(y))$.

Lemma 6.5. Let $|A|>1$. If $t \precsim \mathrm{WIF} u$, then $\mathcal{W} \mathcal{T}(t)=\mathcal{W} \mathcal{T}(u)$.

Proof. It is not hard to see (by substituting $\mathbf{0}$ for all variables in $t$ and $u$ ) that $t \precsim$ WIF $u$ implies that $t$ and $u$ must have the same weak traces ending with an action.

Pick distinct actions $a, b \in A$ and an injection $\iota: V \rightarrow \mathbb{Z}_{>0}$. Let $m=\operatorname{depth}_{w}(u)+1$. Define the closed substitution $\rho$ by $\rho(z)=a^{\iota(z) \cdot m} b \mathbf{0}$ for all $z \in V$. Since $t \precsim$ WIF $u$, we have $\mathcal{W} \mathcal{T}(\rho(t))=\mathcal{W} \mathcal{T}(\rho(u))$. As $m>\operatorname{depth}_{w}(t)=\operatorname{depth}_{w}(u), \iota$ is an injection, and $a$ and $b$ are distinct, it is not hard to see, by Remark 6.4, that this implies $\mathcal{W} \mathcal{T}_{V}(t)=\mathcal{W} \mathcal{T}_{V}(u)$.

Lemma 6.6. Let $|A|>1$. If $t \precsim \mathrm{WIF} u$ and $t \Rightarrow \stackrel{\tau}{\rightarrow} t^{\prime}$, then there is a term $u^{\prime}$ with $u \Rightarrow \stackrel{\tau}{\rightarrow} u^{\prime}$ and $\mathcal{W} \mathcal{T}_{V}\left(u^{\prime}\right) \subseteq \mathcal{W} \mathcal{T}_{V}\left(t^{\prime}\right)$

Proof. Define the closed substitution $\rho$ as in the previous proof. Since $t \precsim$ WIF $u$ and $\rho(t) \Rightarrow \rho\left(t^{\prime}\right)$, there must be a closed term $q$ with $\rho(u) \Rightarrow q$ and $\mathcal{W} \mathcal{T}(q) \subseteq \mathcal{W} \mathcal{T}\left(\rho\left(t^{\prime}\right)\right)$. Since $\rho(z)$ is a $\operatorname{BCCSP}(A)$ term for all $z \in V$, it follows that there is a term $u^{\prime}$ with $u \Rightarrow u^{\prime}$ and $\rho\left(u^{\prime}\right)=q$. Again, as $m>\operatorname{depth}_{w}(u), \iota$ is an injection, and $a$ and $b$ are distinct, it is not hard to see that $\mathcal{W} \mathcal{T}\left(\rho\left(u^{\prime}\right)\right) \subseteq \mathcal{W} \mathcal{T}\left(\rho\left(t^{\prime}\right)\right)$ implies $\mathcal{W} \mathcal{T}_{V}\left(u^{\prime}\right) \subseteq \mathcal{W} \mathcal{T}_{V}\left(t^{\prime}\right)$. In case $u \Rightarrow \stackrel{\tau}{\rightarrow} u^{\prime}$ we are done, so assume $u^{\prime}=u$. Since $t \precsim$ WIF $u$ and $t \stackrel{\tau}{\rightarrow}$, clearly $u \stackrel{\tau}{\rightarrow} u^{\prime \prime}$ for some $u^{\prime \prime}$. And $\mathcal{W} \mathcal{T}_{V}\left(u^{\prime \prime}\right) \subseteq \mathcal{W} \mathcal{T}_{V}(u)=\mathcal{W} \mathcal{T}_{V}\left(u^{\prime}\right) \subseteq \mathcal{W} \mathcal{T}_{V}\left(t^{\prime}\right)$ 
Lemma 6.7. Let $1<|A|<\infty$. Suppose that for some terms $t, u$, substitution $\sigma$, action $a$ and $m>0$ :

(1) $t \precsim$ WIF $u$;

(2) $m \geq \operatorname{depth}_{w}(u)$; and

(3) $\sigma(t) \Rightarrow \stackrel{\tau}{\rightarrow} \hat{t}$ for a term $\hat{t}$ without weak traces $x$ and $a^{m} b$ for any $b \in A$.

Then $\sigma(u) \Rightarrow \stackrel{\tau}{\rightarrow} \hat{u}$ for a term $\hat{u}$ without weak traces $x$ and $a^{m} b$ for any $b \in A$.

Proof. Based on proviso (3) there are two cases to consider.

- $t \Rightarrow \stackrel{\tau}{\rightarrow} t^{\prime}$ for some term $t^{\prime}$ with $\sigma\left(t^{\prime}\right)=\hat{t}$. By Lem. 6.6 there is a term $u^{\prime}$ with $u \Rightarrow \stackrel{\tau}{\rightarrow} u^{\prime}$ and $\mathcal{W} \mathcal{T}_{V}\left(u^{\prime}\right) \subseteq \mathcal{W} \mathcal{T}_{V}\left(t^{\prime}\right)$. So $\sigma(u) \Rightarrow \stackrel{\tau}{\rightarrow} \sigma\left(u^{\prime}\right)$. By proviso $(3)$ of the lemma, $x \notin$ $\mathcal{W} \mathcal{T}_{V}\left(\sigma\left(t^{\prime}\right)\right) \supseteq \mathcal{W} \mathcal{T}_{V}\left(\sigma\left(u^{\prime}\right)\right)$. Assume, toward a contradiction, that $a^{m} b \in \mathcal{W} \mathcal{T}\left(\sigma\left(u^{\prime}\right)\right)$ for some $b \in A$. Since $m \geq \operatorname{depth}_{w}(u)$, clearly there is a $k \leq m$ and a $y \in V$ such that $a^{k} y \in \mathcal{W} \mathcal{T}_{V}\left(u^{\prime}\right)$ and $a^{m-k} b \in \mathcal{W} \mathcal{T}(\sigma(y))$. Since $a^{k} y \in \mathcal{W} \mathcal{T}_{V}\left(u^{\prime}\right) \subseteq \mathcal{W} \mathcal{T}_{V}\left(t^{\prime}\right)$, it follows that $a^{m} b \in \mathcal{W} \mathcal{T}\left(\sigma\left(t^{\prime}\right)\right)$, which contradicts proviso $(3)$ of the lemma. Hence we can take $\hat{u}=\sigma\left(u^{\prime}\right)$.

- $y \in \mathcal{W} \mathcal{T}_{V}(t)$ and $\sigma(y) \Rightarrow \stackrel{\tau}{\rightarrow} \hat{t}$ for some $y \in V$. Lem. 6.5 yields $y \in \mathcal{W} \mathcal{T}_{V}(u)$, so $\sigma(u) \Rightarrow \stackrel{\tau}{\rightarrow} \hat{t}$. Hence we can take $\hat{u}=\hat{t}$.

Proposition 6.8. Let $1<|A|<\infty$, and let the axiomatization $E$ be sound for $\operatorname{BCCS}(A)$ modulo $\precsim$ WIF. Suppose that for some terms $v, w$, action $a$ and $m>0$ :

(1) $E \vdash v \preccurlyeq w$;

(2) $m \geq \max \left\{\operatorname{depth}_{w}(u) \mid t \preccurlyeq u \in E\right\}$; and

(3) $v \Rightarrow \stackrel{\tau}{\rightarrow} \hat{v}$ for a term $\hat{v}$ without weak traces $x$ and $a^{m} b$ for any $b \in A$.

Then $w \Rightarrow \stackrel{\tau}{\rightarrow} \hat{w}$ for a term $\hat{w}$ without weak traces $x$ and $a^{m} b$ for any $b \in A$.

Proof. By induction on a derivation of $E \vdash v \preccurlyeq w$. Reflexivity is trivial; we focus on the other cases.

- Suppose $E \vdash v \preccurlyeq w$ because $\sigma(t)=v$ and $\sigma(u)=w$ for some $t \preccurlyeq u \in E$ and substitution $\sigma$. The claim then follows by Lem. 6.7.

- Suppose $E \vdash v \preccurlyeq w$ because $E \vdash v \preccurlyeq u$ and $E \vdash u \preccurlyeq w$ for some $u$. By induction, $u \Rightarrow \stackrel{\tau}{\rightarrow} \hat{u}$ for a term $\hat{u}$ without weak traces $x$ and $a^{m} b$ for any $b \in A$.. Hence, again by induction, $w \Rightarrow \stackrel{\tau}{\rightarrow} \hat{w}$ for a term $\hat{w}$ without weak traces $x$ and $a^{m} b$ for any $b \in A$.

- Suppose $E \vdash v \preccurlyeq w$ because $v=v_{1}+v_{2}$ and $w=w_{1}+w_{2}$ with $E \vdash v_{1} \preccurlyeq w_{1}$ and $E \vdash v_{2} \preccurlyeq w_{2}$. Since $v \Rightarrow \stackrel{\tau}{\rightarrow} \hat{v}$, we have $v_{1} \Rightarrow \stackrel{\tau}{\rightarrow} \hat{v}$ or $v_{2} \Rightarrow \stackrel{\tau}{\rightarrow} \hat{v}$. Assume, without loss of generality, that $v_{1} \Rightarrow \stackrel{\tau}{\rightarrow} \hat{v}$. By induction, $w_{1} \Rightarrow \stackrel{\tau}{\rightarrow} \hat{w}$ for a term $\hat{w}$ without weak traces $x$ and $a^{m} b$ for any $b \in A$. And $w \Rightarrow \stackrel{\tau}{\rightarrow} \hat{w}$.

- Suppose $E \vdash v \preccurlyeq w$ because $v=\alpha v_{1}$ and $w=\alpha w_{1}$ with $E \vdash v_{1} \preccurlyeq w_{1}$. By proviso (3) of the proposition, $\alpha=\tau$, and either $v_{1}=\hat{v}$ or $v_{1} \Rightarrow \stackrel{\tau}{\rightarrow} \hat{v}$. In the first case, by proviso (3) of the proposition and Lem. 6.5, $w_{1}$ has no weak traces $x$ and $a^{m} b$ for any $b \in A$. And $w \stackrel{\tau}{\rightarrow} w_{1}$. In the second case, by induction, $w_{1} \Rightarrow \stackrel{\tau}{\rightarrow} \hat{w}$ for a term $\hat{w}$ without weak traces $x$ and $a^{m} b$ for any $b \in A$. And $w \Rightarrow \stackrel{\tau}{\rightarrow} \hat{w}$. 
Theorem 6.9. If $1<|A|<\infty$, then the inequational theory of $B C C S(A)$ modulo $\precsim \mathrm{WIF}$ does not have a finite basis.

Proof. Let $E$ be a finite axiomatization over $\operatorname{BCCS}(A)$ that is sound modulo $\precsim$ wIF. Let $m$ be greater than the weak depth of any term in $E$. According to Prop. 6.8, the inequation $\tau\left(a^{m} x\right)+\Phi_{m} \preccurlyeq \Phi_{m}$ cannot be derived from $E$. Yet it is sound modulo $\precsim$ WIF.

Likewise, the inequational theory of $\operatorname{BCCSP}(A)$ modulo $\precsim_{\text {IF }}$ also does not have a finite basis in case of a non-singleton finite alphabet. If $A$ has two distinct elements $a$ and $b$, then the cornerstone for this negative result is the following infinite family of inequations, for each $m \geq 0$ :

$$
a^{m+1} x+\Psi_{m} \preccurlyeq \Psi_{m}
$$

with

$$
\Psi_{m}=a\left(a^{m} x+x\right)+\sum_{b \in A} a\left(a^{m} x+a^{m} b \mathbf{0}\right)
$$

The proof of the following theorem, which can be found in [CF08, is very similar to the proof of Thm. 6.9, and is therefore omitted here.

Theorem 6.10. If $1<|A|<\infty$, then the inequational theory of BCCSP(A) modulo $\precsim$ IF does not have a finite basis.

6.3.2. $|A|=1$. We prove that the inequational theory of $\operatorname{BCCS}(A)$ modulo $\precsim$ wIF does not have a finite basis in case of a singleton alphabet. The cornerstone for this negative result is the following infinite family of inequations, for each $m \geq 0$ :

$$
a^{m} x \preccurlyeq a^{m} x+x
$$

If $|A|=1$, then these inequations are clearly sound modulo $\precsim$ WIF. In particular, for any closed substitution $\rho, \mathcal{W} \mathcal{T}(\rho(x)) \subseteq \mathcal{W} \mathcal{T}\left(\rho\left(a^{m} x\right)\right)$.

For $m \geq 1$, the inequations above show that Lem. 6.5 fails if $|A|=1$. We now formulate a weaker variant of this lemma for $|A|=1$.

Lemma 6.11. Let $A=\{a\}$. If $t \precsim$ WIF $u$, then $\mathcal{W} \mathcal{T}_{V}(t) \subseteq \mathcal{W} \mathcal{T}_{V}(u)$.

Proof. Select an injection $\iota: V \rightarrow \mathbb{Z}_{>0}$. Let $m=\operatorname{depth}_{w}(u)+1$. Define the closed substitution $\rho$ by $\rho(z)=a^{\iota(z) \cdot m} \mathbf{0}$ for all $z \in V$. Since $t \precsim$ WIF $u$, we have $\mathcal{W} \mathcal{T}(\rho(t))=\mathcal{W} \mathcal{T}(\rho(u))$. Consider any $a^{k} y \in \mathcal{W T}_{V}(t)$. Then $a^{k+\iota(y) \cdot m} \in \mathcal{W} \mathcal{T}(\rho(t))=\mathcal{W} \mathcal{T}(\rho(u))$. As $m>\operatorname{depth}_{w}(u) \geq k$ and $\iota$ is an injection, it is not hard to see that this implies $a^{k} y \in \mathcal{W} \mathcal{T}_{V}(u)$.

Lemma 6.12. Let $A=\{a\}$. Suppose that for some terms $t, u$, substitution $\sigma$, variable $x$ and $m>0$ :

(1) $t \precsim$ WIF $u$;

(2) $m>\operatorname{depth}_{w}(u)$; and

(3) $x \in \mathcal{W} \mathcal{T}_{V}(\sigma(u))$ and $a^{k} x \notin \mathcal{W} \mathcal{T}_{V}(\sigma(u))$ for all $1 \leq k<m$.

Then $x \in \mathcal{W} \mathcal{T}_{V}(\sigma(t))$ and $a^{k} x \notin \mathcal{W} \mathcal{T}_{V}(\sigma(t))$ for all $1 \leq k<m$.

Proof. Since $x \in \mathcal{W} \mathcal{T}_{V}(\sigma(u))$, clearly there is a $y \in \mathcal{W} \mathcal{T}_{V}(u)$ with $x \in \mathcal{W} \mathcal{T}_{V}(\sigma(y))$. Consider the closed substitution $\rho$ defined by $\rho(y)=a^{m} \mathbf{0}$ and $\rho(z)=\mathbf{0}$ for all $z \neq y$. Since $y \in \mathcal{W} \mathcal{T}_{V}(u)$, we have $a^{m} \in \mathcal{W} \mathcal{T}(\rho(u))$. So $t \precsim$ WIF $u$ implies $a^{m} \in \mathcal{W} \mathcal{T}(\rho(t))$. Since $m>\operatorname{depth}_{w}(u)=\operatorname{depth}_{w}(t)$, clearly there are some $\ell<m$ and $z \in V$ such that 
$a^{\ell} z \in \mathcal{W} \mathcal{T}_{V}(t)$ and $a^{m-\ell} \in \mathcal{W} \mathcal{T}(\rho(z))$. As $\ell<m$, it follows from the definition of $\rho$ that $z=y$. Since $a^{\ell} y \in \mathcal{W} \mathcal{T}_{V}(t)$ and $x \in \mathcal{W} \mathcal{T}_{V}(\sigma(y))$, it follows that $a^{\ell} x \in \mathcal{W} \mathcal{T}_{V}(\sigma(t))$. By Lem. 6.11, $a^{k} x \notin \mathcal{W} \mathcal{T}_{V}(\sigma(t))$ for all $1 \leq k<m$. Hence we obtain $\ell=0$.

Proposition 6.13. Let $A=\{a\}$, and let the axiomatization $E$ be sound for $\operatorname{BCCS}(A)$ modulo $\precsim$ WIF. Suppose that for some terms $v, w$, variable $x$ and $m>0$ :

(1) $E \vdash v \preccurlyeq w$;

(2) $m>\max \left\{\operatorname{depth}_{w}(u) \mid t \preccurlyeq u \in E\right\}$; and

(3) $x \in \mathcal{W} \mathcal{T}_{V}(w)$ and $a^{k} x \notin \mathcal{W} \mathcal{T}_{V}(w)$ for all $1 \leq k<m$.

Then $x \in \mathcal{W} \mathcal{T}_{V}(v)$ and $a^{k} x \notin \mathcal{W} \mathcal{T}_{V}(v)$ for all $1 \leq k<m$.

Proof. By induction on a derivation of $E \vdash v \preccurlyeq w$. Reflexivity is trivial; we focus on the other cases.

- Suppose $E \vdash v \preccurlyeq w$ because $\sigma(t)=v$ and $\sigma(u)=w$ for some $t \preccurlyeq u \in E$ and substitution $\sigma$. The claim then follows by Lem. 6.12.

- Suppose $E \vdash v \preccurlyeq w$ because $E \vdash v \preccurlyeq u$ and $E \vdash u \preccurlyeq w$ for some $u$. By induction, $x \in \mathcal{W} \mathcal{T}_{V}(u)$ and $a^{k} x \notin \mathcal{W} \mathcal{T}_{V}(u)$ for all $1 \leq k<m$. Hence, again by induction, $x \in \mathcal{W} \mathcal{T}_{V}(v)$ and $a^{k} x \notin \mathcal{W} \mathcal{T}_{V}(v)$ for all $1 \leq k<m$.

- Suppose $E \vdash v \preccurlyeq w$ because $v=v_{1}+v_{2}$ and $w=w_{1}+w_{2}$ with $E \vdash v_{1} \preccurlyeq w_{1}$ and $E \vdash v_{2} \preccurlyeq w_{2}$. Since $x \in \mathcal{W} \mathcal{T}_{V}(w)$, we have $x \in \mathcal{W} \mathcal{T}_{V}\left(w_{1}\right)$ or $x \in \mathcal{W} \mathcal{T}_{V}\left(w_{2}\right)$. Assume, without loss of generality, that $x \in \mathcal{W} \mathcal{T}_{V}\left(w_{1}\right)$. Since $a^{k} x \notin \mathcal{W} \mathcal{T}_{V}(w)$ for all $1 \leq k<m$, surely $a^{k} x \notin \mathcal{W} \mathcal{T}_{V}\left(w_{1}\right)$ for all $1 \leq k<m$. By induction, $x \in \mathcal{W} \mathcal{T}_{V}\left(v_{1}\right)$, and hence $x \in \mathcal{W} \mathcal{T}_{V}(v)$. For all $1 \leq k<m$ we have $a^{k} x \notin \mathcal{W} \mathcal{T}_{V}(w)$, and hence $a^{k} x \notin \mathcal{W} \mathcal{T}_{V}(v)$, by Lem. 6.11.

- Suppose $E \vdash v \preccurlyeq w$ because $v=\alpha v_{1}$ and $w=\alpha w_{1}$ with $E \vdash v_{1} \preccurlyeq w_{1}$. By proviso (3) of the proposition, $\alpha=\tau, x \in \mathcal{W} \mathcal{T}_{V}\left(w_{1}\right)$ and $a^{k} x \notin \mathcal{W} \mathcal{T}_{V}\left(w_{1}\right)$ for all $1 \leq k<m$. By induction, $x \in \mathcal{W} \mathcal{T}_{V}\left(v_{1}\right)$ and $a^{k} x \notin \mathcal{W} \mathcal{T}_{V}\left(v_{1}\right)$ for all $1 \leq k<m$. Hence $x \in \mathcal{W} \mathcal{T}_{V}(v)$ and $a^{k} x \notin \mathcal{W} \mathcal{T}_{V}(v)$ for all $1 \leq k<m$.

Theorem 6.14. If $|A|=1$, then the inequational theory of $B C C S(A)$ modulo $\precsim \mathrm{WIF}$ does not have a finite basis.

Proof. Let $E$ be a finite axiomatization over $\operatorname{BCCS}(A)$ that is sound modulo $\precsim$ wIF. Let $m$ be greater than the weak depth of any term in $E$. According to Prop. 6.13, the inequation $a^{m} x \preccurlyeq a^{m} x+x$ cannot be derived from $E$. Yet, since $|A|=1$, it is sound modulo $\precsim$ WIF.

Likewise, the inequational theory of $\operatorname{BCCSP}(A)$ modulo $\precsim_{\text {IF }}$ does not have a finite basis in case of a singleton alphabet. This negative result is based on the same infinite family of inequations as for the weak case: $a^{m} x \preccurlyeq a^{m} x+x$ for each $m \geq 0$. The proof of the following theorem is more or less identical to the proof of Thm. 6.14, and is therefore omitted here.

Theorem 6.15. If $|A|=1$, then the inequational theory of $B C C S P(A)$ modulo $\precsim$ IF does not have a finite basis. 


\section{Conclusion}

We have introduced a method to transform an axiomatization for a concrete semantics in the context of the process algebra BCCSP to an axiomatization for a corresponding weak semantics with regard to BCCS, such that the properties ground-completeness and $\omega$-completeness are preserved. Trace, (a form of) completed trace, failures and impossible futures semantics are within the realm of this transformation method.

Exploiting this approach, we obtained axiomatizations for the weak trace, completed trace and failures preorders and equivalences. Moreover, we performed a comprehensive and systematic study on the axiomatizability of concrete and weak impossible futures semantics over BCCSP and BCCS. Table 1 presents an overview, where + indicates that a finite axiomatization exists, while - indicates that a finite axiomatization does not exist. The table expands in two dimensions: ground-completeness vs. $\omega$-completeness and preorder vs. equivalence. When necessary, we distinguish two categories, according to the cardinality of the alphabet $A$ : finite or infinite.

\begin{tabular}{|c|c|c|c|}
\hline \multirow{2}{*}{} & ground-completeness & \multicolumn{2}{|c|}{$\omega$-completeness } \\
\cline { 2 - 4 } & $1 \leq|A| \leq \infty$ & $|A|=\infty$ & $1 \leq|A|<\infty$ \\
\hline (concrete or weak) imp. futures preorder & + & + & - \\
\hline (concrete or weak) imp. futures equivalence & - & - & - \\
\hline
\end{tabular}

Table 1: Summary of results for impossible futures semantics

Impossible futures semantics is the first example that, in the context of BCCSP/BCCS, affords a ground-complete axiomatization modulo the preorder, while missing a groundcomplete axiomatization modulo the equivalence.

Acknowledgement. This research was initiated by questions from Jos Baeten, on axiomatizing impossible futures semantics, and from David de Frutos-Escrig, on relating the axiomatizability of concrete and weak impossible futures semantics.

\section{REFERENCES}

[AFGI04] L. Aceto, W. Fokkink, R.J. van Glabbeek and A. Ingolfsdottir. Nested semantics over finite trees are equationally hard. Inf. Comput., 191(2):203-232, 2004.

[AFI07] L. Aceto, W. Fokkink and A. Ingolfsdottir. Ready to preorder: Get your BCCSP axiomatization for free! In Proc. CALCO'0\%, LNCS 4624, pp. 65-79, Springer, 2007.

[AFIL05] L. Aceto, W. Fokkink, A. Ingolfsdottir and B. Luttik. Finite equational bases in process algebra: Results and open questions. In Processes, Terms and Cycles: Steps on the Road to Infinity, LNCS 3838, pp. 338-367, Springer, 2005.

[BHR84] S.D. Brookes, C.A.R. Hoare and A.W. Roscoe. A theory of communicating sequential processes. J. ACM, 31(3):560-599, 1984.

[CF08] T. Chen and W. Fokkink. On the axiomatizability of impossible futures: Preorder versus equivalence. In Proc. LICS'08, IEEE, pp. 156-165, 2008.

[CFG08] T. Chen, W. Fokkink and R.J. van Glabbeek. Ready to preorder: The case of weak process semantics. Inf. Proc. Lett., 109(2):104-111, 2008.

[CFG09] T. Chen, W. Fokkink and R.J. van Glabbeek. On finite bases for weak semantics: Failures versus impossible futures. In Proc. SOFSEM'09, LNCS 5404, pp. 167-180, Springer, 2009.

[CFLN08] T. Chen, W. Fokkink, B. Luttik and S. Nain. On finite alphabets and infinite bases. Inf. Comput. 206(5):492-519, 2008. 
[DH84] R. De Nicola and M. Hennessy. Testing equivalences for processes. Theo. Comp. Sci. 34, pp. 83-133, 1984.

[FN05] W.J. Fokkink and S. Nain. A finite basis for failure semantics. In Proc. ICALP'05, LNCS 3580, pp. 755-765, Springer, 2005.

[FGP07] D. de Frutos-Escrig, C. Gregorio-Rodríguez and M. Palomino. Ready to preorder: An algebraic and general proof. J. Log. Algebr. Program, 78(7):539-551, 2009.

[G93a] R.J. van Glabbeek. The linear time - branching time spectrum II. The semantics of sequential systems with silent moves. In Proc. CONCUR'93, LNCS 715, Springer, pp. 66-81, 1993.

[G93b] R.J. van Glabbeek. A complete axiomatization for branching bisimulation congruence of finitestate behaviours. In Proc. MFCS'93, LNCS 711, pp. 473-484, 1993.

[G97] R.J. van Glabbeek. Notes on the methodology of CCS and CSP. Theo. Comp. Sci. 177(2):329-349. 1997.

[G01] R.J. van Glabbeek. The linear time - branching time spectrum I. The semantics of concrete, sequential processes. In Handbook of Process Algebra, pp. 3-99, Elsevier, 2001.

[G05] R.J. van Glabbeek. A characterisation of weak bisimulation congruence. In Processes, Terms and Cycles: Steps on the Road to Infinity, LNCS 3838, pp. 26-39, Springer, 2005.

[GV06] R.J. van Glabbeek and M. Voorhoeve. Liveness, fairness and impossible futures. In Proc. CONCUR'06, LNCS 4137, pp. 126-141, Springer, 2006.

[G90] J.F. Groote. A new strategy for proving $\omega$-completeness with applications in process algebra. In Proc. CONCUR'90, LNCS 458, pp. 314-331, Springer, 1990.

[M89] R. Milner. A complete axiomatisation for observational congruence of finite-state behaviours. Inf. Comput. 81:227-247, 1989

[RV07] A. Rensink and W. Vogler. Fair testing. Inf. Comput., 205(2):125-198, 2007.

[RB81] W. Rounds and S. Brookes. Possible futures, acceptances, refusals and communicating processes. In Proc. FOCS'81, pp. 140-149. IEEE, 1981.

[V92] W. Vogler. Modular Construction and Partial Order Semantics of Petri Nets. LNCS 625, Springer, 1992.

[VM01] M. Voorhoeve and S. Mauw. Impossible futures and determinism. Inf. Proc. Lett., 80(1):51-58, 2001.

This work is licensed under the Creative Commons Attribution-NoDerivs License. To view a copy of this license, visit http://creativecommons.org/licenses/by-nd/2.0/ or send a letter to Creative Commons, 171 Second St, Suite 300, San Francisco, CA 94105, USA, or Eisenacher Strasse 2, 10777 Berlin, Germany 